\title{
Adaptive Fingerprinting in Multi-Sensor Fusion for Accurate Indoor Tracking
}

\author{
Alberto Belmonte-Hernández, Gustavo Hernández-Peñaloza, Federico Álvarez, Giuseppe Conti
}

\begin{abstract}
Indoor Localization and Tracking have become an attractive research topic because of the wide range of potential applications. These applications are highly demanding in terms of estimation accuracy and rise a challenge due to the complexity of the scenarios modeled. Approaches for these topics are mainly based on either deterministic or probabilistic methods such as Kalman or Particles Filter. These techniques are improved by fusing information from different sources such as wireless or optical sensors. In this paper, a novel MUlti-sensor Fusion using Adaptive Fingerprint (MUFAF) Algorithm is presented and compared with several multi-sensor indoor localization and tracking methods. MUFAF is mainly divided in four phases: first, a Target Position Estimation (TPE) process is performed by every sensor; second, a Target Tracking Process (TTP) stage; third, a Multi-Sensor fusion (MMF) combines the sensor information and finally, an Adaptive Fingerprint Update (AFU) is applied. For TPE, a complete environment characterization in combination with a Kernel Density Estimation (KDE) technique are employed to obtain object position. A Modified Kalman Filter (MKF) is applied to TPE output in order to smooth target routes and avoid outliers effect. Moreover, two fusion methods are described in this work: Track-To-Track Fusion (TTTF) and Kalman Sensor Group Fusion (KSGF). Finally, AFU will endow the algorithm with responsiveness to environment changes by using Kriging interpolation to update the scenario fingerprint. MUFAF is implemented and compared in a testbed showing that it provides a significant improvement in estimation accuracy and long-term adaptivity to condition changes.
\end{abstract}

Index Terms-Wireless, Receive Signal Strength Indicator (RSSI), Indoor Tracking, Kernel Density Estimation, Kalman filter, Multi-Sensor Fusion.

\section{INTRODUCTION}

Indoor positioning and tracking have attracted an extensive research effort because of their usefulness for a broad range of applications such as audience pattern generation [1], costumer analysis in retail [2], surveillance [3], business related activities [4], healthcare behavioral monitoring [5] among others [6], [7].

The aim is mainly to extract location and routes from indoor targets to provide applications which make use of both estimated values. One approach is the use of cameras [3], [8], but cost, occlusions, poor/inadequate lighting conditions or privacy issues limit their applicability in several scenarios. In this paper we will focus on a very common approach which is the use of a sensors deployment to detect and track the objects, however the precision in the location and tracking is key to the applications using such data.

Recent research works face this problem by implementing wireless sensors in combination with processing algorithms that combine data gathered from multiple nodes [2], [6], [7].
Within the methods to estimate indoor position, most popular are Time-of-Arrival (ToA) [9], Time Difference of Arrival (TDoA) [10], Angle-of-Arrival (AoA) [11] and Received Signal Strength Indicator (RSSI) measurements [12], being the techniques more often employed ToA and RSSI. On the one hand, ToA techniques use timestamps lags from a sent/received packet. These methods allow high precision in the final estimation with relative low background processing. However, hardware cost limitations in addition to strict synchronization requirements increase the system complexity making this approach hard to implement. On the other hand, RSSI values can be measured by a large number of wireless devices but RSSI modeling is generally tedious due to the harsh propagation conditions and processing techniques to achieve a good accuracy [18].

Because of the high potentiality of indoor tracking applications, low cost and the wide range of devices that can be used, the technical approach presented in this work relies on RSSI-based estimation.

RSSI position estimation works have been presented in the literature for indoor tracking [13], [14], [15], [16], employing theoretical propagation models to estimate the distance and/or in combination with deterministic methods for tracking [14]. However, these propagation models neglect the obstacles, which yields to a high error rate in estimations.

To correct and improve such problem with obstacles, a very extended approach is based on the employment of Fingerprinting technique which characterizes the scenario by splitting into cells and performing spatial sampling [13], [14] with the aim of modeling the signal propagation in the grid. This technique can be applied for both deterministic and probabilistic methods. Deterministic methods depend heavily on the resolution of the fingerprint cells: the shorter the number of cells is, the lower the estimation accuracy is. Probabilistic methods consider a few sensors introducing a high mathematical treatment to fuse the information gathered [15], [16] and in this way result get improved.

Additionally, recent works incorporate an adaptive update stage to improve the algorithmic accuracy [17], [19] which is a growing research topic as it allows to adjust fingerprint-based technology to changes in environment conditions.

Therefore, following this line to improve the accuracy and motivated by the heavy estimation requirements in terms of precision for indoor tracking, and considering the large availability of commercial devices (e.g. smartphones) with several sensors (wireless communication standards) in this work a MUlti- Sensor Fusion based on Adaptive Fingerprint (MUFAF) Algorithm is presented to perform the object track- 
ing (in this paper we used IEEE 802.15.1, IEEE 802.15.4 and IEEE 802.11) based on RSSI measurements.

To achieve the presented aims, that in the paper are compared to other approaches, MUFAF works as follows. MUFAF starts by performing a statistical position estimation called Target Position Estimation (TPE) in each of the available sensors (measuring in the different wireless interfaces). A complete environment characterization in combination with a Kernel Density Estimation (KDE) technique are employed to obtain object position. and a Modified Kalman Filter (MKF) is applied to TPE output in order to smooth target routes and avoid outliers effect.

Once data is captured, a Target Tracking Process (TTP) is done to obtain the object routes from individual sensors. Subsequently, the fusion of the sensors information is processed by a Multi-Sensor fusion (MMF) process which combines the sensor data using Track-To-Track Fusion (TTTF) and/or Kalman Sensor Group Fusion (KSGF), which are described and compared in the paper.

Last step is to provide an Adaptive Fingerprint Update (AFU) to cope with the environment changes. AFU uses a novel adaptive fingerprint update technique to adjust the algorithm according to scenario variations based on Kriging interpolation.

The remainder of this paper is organized as follows: In next section II, the problem statement, notation and assumptions are addressed. III, Target Position Estimation (TPE) is detailed. Section IV describes the Target Tracking Procedure (TTP). In section V, the comparison of fusion pattern architectures is defined. In section VI adaptive fingerprinting technique using Kriging is presented. Moreover, in section VII the implementation details and results of the algorithms are illustrated. Finally, conclusions and future work are drawn in section VIII.

\section{PROBLEM STATEMENT}

Complex indoor environments have been considered, where harsh signal propagation conditions present a challenge for appropriate characterization. For this aiming, it is assumed an indoor scenario with a set $\mathbf{v}=\left\{v_{i, \lambda} \mid i=1, \ldots, I ; \lambda=\right.$ $1, \ldots, \Lambda\}$ of Access Points (AP) located at Cartesian coordinates $\mathbf{p}_{i}=\left(x_{i}, y_{i}\right) \in \mathbb{R}^{2}$. Furthermore, let $\Lambda$ be the number of sensors that each node is equipped with. In this work, four sensors from three wireless technologies are considered: IEEE802.11.g/WI-FI [53], IEEE802.15.1/Bluetooth v4.0 [51], IEEE802.15.4/XBee and IEEE802.15.4/CC2420 [52]. Network nodes are able to obtain the Received Signal Strength Indicator, denoted by RSS, from the monitored target. Consequently, $R S S_{i, \lambda}$ describes the RSSI measurements gathered by node $i$ using technology $\lambda$.

The main problems addressed in this work are three: $(a)$ the accurate localization estimation of the target by means of a combination of available RSSI measurements sensed by the nodes; $(b)$ tracking of the detected object in an indoor environment and $(c)$ the improvement in trajectories accuracy by means of multi-sensor fusion of the different technologies considered. To tackle these challenges, several considerations are taken:
TABLE I

MUFAF NOTATION

\begin{tabular}{|c|c|}
\hline & $\begin{array}{l}\text { Fingerprint and Target Position Estima- } \\
\text { tion (TPE): }\end{array}$ \\
\hline$\lambda$ & a wireless sensor technology. \\
\hline $\mathbf{v}$ & set of nodes in the network. \\
\hline$\hat{\mathbf{p}}_{x, y}^{\lambda}$ & $\begin{array}{l}\text { Estimated position for a particular technol- } \\
\text { ogy. }\end{array}$ \\
\hline $\mathbf{v}_{\mathbf{p}_{x, y}, \lambda}$ & general definition of a node. \\
\hline $\mathbf{R S S}_{\mathbf{p}, \lambda}$ & $\begin{array}{l}\text { Set of RSSI measurements gathered by a } \\
\text { node using a particular technology. }\end{array}$ \\
\hline$c=\left\{\mathbf{p}_{l} \mid 1, \ldots, L\right\}$ & set of fingerprint cells \\
\hline & window time for fingerprinting in a cell. \\
\hline$K(\cdot) ; h$ & Kernel Function and bandwidth. \\
\hline$d\left(\mathbf{p}_{1}, \mathbf{p}_{2}\right)$ & Euclidean distance from $\mathbf{p}_{1}$ to $\mathbf{p}_{2}$. \\
\hline $\mathbf{F}\left(p_{l}, \lambda, R S S\right)$ & $\begin{array}{l}\text { Fingerprint Matrix. } \\
\text { Target tracking Process (TTP): }\end{array}$ \\
\hline$k$ & Time step for MUFAF iteration. \\
\hline $\mathbf{x}$ & system state for Kalman Filter. \\
\hline $\mathbf{A}$ & Kalman Transition Matrix. \\
\hline$u$ & System input (Random Walk Model RWM). \\
\hline B & Kalman Control Matrix (RWM). \\
\hline G & Kalman Gain Matrix. \\
\hline $\mathbf{H}$ & Measurements Prediction. \\
\hline $\mathrm{Q}$ & Error covariance using RWM. \\
\hline $\mathbf{R}$ & Error covariance matrix from Observations. \\
\hline$\eta$ & Noise Process. \\
\hline $\begin{array}{l}\hat{\mathbf{p}}_{x, y} \\
\mathbf{z}=\hat{\mathbf{p}}_{x, y}\end{array}$ & $\begin{array}{l}\text { TPP Input: estimated position in TPE, } \\
\text { Observation. }\end{array}$ \\
\hline $\begin{array}{l}p_{x} ; p_{y} \\
\mathbf{P}\end{array}$ & $\begin{array}{l}\text { Cartesian coordinates in axis } x \text { and } y \text {. } \\
\text { Covariance Matrix from Kalman Process. } \\
\text { Multi-Sensor Fusion: }\end{array}$ \\
\hline $\mathfrak{R}^{\lambda}$ & A route from a target tracked. \\
\hline$\varpi$ & Number of steps that compose a route. \\
\hline $\mathfrak{d}$ & Order of $\mathbf{P}$ Matrix. \\
\hline $\mathbf{P}_{\lambda, k}$ & $\begin{array}{l}\text { Covariance Matrix from Kalman process in } \\
\text { a time } k \text { for a technology } \lambda \text {. }\end{array}$ \\
\hline $\bar{p}=\left(x_{m}, y_{m}\right)$ & $\begin{array}{l}\text { position estimated after fusion stage. Addi- } \\
\text { tionally, } x_{m}, y_{m} \text { are the spatial coordinates } \\
\text { employed as input to the alpha-beta filter. }\end{array}$ \\
\hline$\alpha, \beta$ & $\begin{array}{l}\text { control parameters of Alpha-Beta filter. } \\
\text { Adaptive Fingerprint Update (AFU) }\end{array}$ \\
\hline $\mathbf{h}$ & $\begin{array}{l}\text { Set of distances in empirical } \\
\text { Semivariogram. }\end{array}$ \\
\hline$\hat{\gamma}(\cdot)$ & Empirical Semivariogram. \\
\hline $\bar{\gamma}(\cdot)$ & Theoretical Semivariogram. \\
\hline$n g, S i, R g$ & Fitting parameters in $\bar{\gamma}(\cdot)$ \\
\hline$W ; D$ & Width and Depth of a room. \\
\hline & weights in Kriging estimation. \\
\hline$\Gamma$ & Semivariogram Matrix for prediction. \\
\hline $\mathcal{L}$ & Lagrange Multiplier. \\
\hline s & vector containing w and $\mathcal{L}$. \\
\hline b & Similarity measure to estimated location. \\
\hline M & Number of neighbors chosen for AFU. \\
\hline$\mu$ & Decision Threshold for AFU. \\
\hline
\end{tabular}

- RSS is assumed to be an independent and identically distributed (i.i.d.) Random Variable.

- Every node $v_{i, \lambda}$ is able to gather a set of RSS measurements from the aforementioned technologies $\lambda$. Notice that it is possible to obtain $R S S_{i, \lambda} \rightarrow \emptyset$

- Additionally, it is assumed that $\mathbf{R S S}_{I, \lambda}$ is a vector containing synchronized measurements from all nodes $I$ for a particular technology $\lambda$.

- A window-time, denoted by $k$, is considered. Data gathered in this period will be mathematically treated as synchronized. For experiments performed in this paper, $k=1$ sec. There are several factors that can affect this window-time. Firstly, the frequency sampling of the de- 
vices considered in this work is high which allows to have a representative data-set for position estimation. It implies that the window-time can be reduced. However, outliers can decrease the estimation quality as a consequence of overestimation. In concrete, IEEE802.15.4Xbee is able to forward up to 30 packets per second (pps); IEEE802.11.g-Wi-FI frequency sampling is above $60 \mathrm{pps}$ and IEEE802.15.1 Bluetooth is up to 20 pps. Secondly, the velocity of persons moving in the scene is a key parameter with a wide range of values. When a person is running in the scene, a shorter window-time can be appropriated for the position estimation. Nonetheless, the aforementioned problem can arise. Moreover, some approaches quantify an average of a normal person walking to be around $130 \mathrm{cms} /$ second [29], [30]. In tests performed, 1 Second time window provides an optimal trade-off.

In order to maintain general conventions on the techniques presented, an overview of the notation employed in this work is detailed in table I.

\section{A. Main contributions}

The key contribution of this work is the proposal of a complete system for indoor localization and tracking that overcomes the difficult conditions of the scenarios in terms of propagation interferences. Moreover, MUFAF is scalable and modular. Experiments performed have shown that the algorithms described are able to track several objects simultaneously. Furthermore, MUFAF outperforms existing techniques therefore it can be considered as an adaptive system fusing multiple data sources. The main technical contributions of this paper are:

- Several multi-sensor architecture patterns for tracking systems are compared. Some of the most precise methods for indoor tracking are described and assessed in this work. As a result, MUFAF Algorithm for indoor localization using multi-sensor fusion from various technologies.

- An Adaptive Fingerprint Update (AFU) procedure is proposed to ensure that MUFAF will change according to the varying scenario conditions. This algorithm is based on Kriging interpolation that is a statistical technique that does not depend on the field mean. This feature guarantees AFU stage to be unbiased.

- A study on indoor localization and tracking is provided. As a result, a complete open dataset is presented. This dataset contains all required fingerprinting measurements for the technologies considered. Additionally, it also comprises the route measurements to allow scientific community to test and compare their algorithms with the ones presented in this work.

\section{Position Estimation}

In this section, the algorithm to obtain the object position in an indoor environment is described. This process comprises two steps: (a) Fingerprinting and $(b)$ Kernel Density Estimation (KDE). The former is a method for scenario characterization while the latter is a statistical technique for

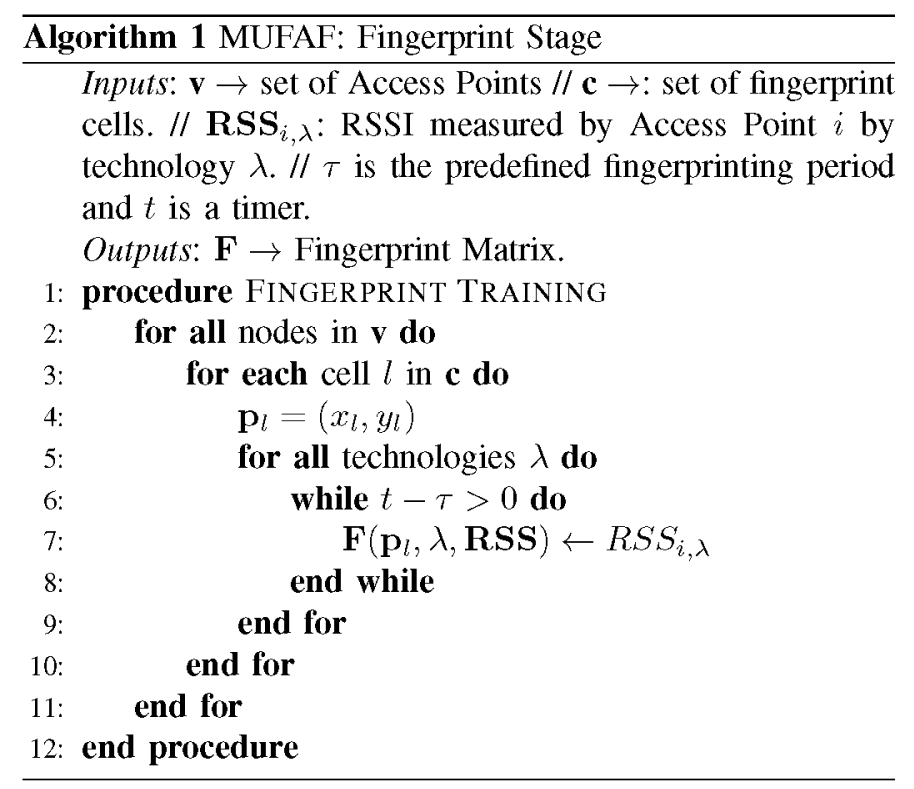

position estimation applied to sensor RSSI measurements. $\mathrm{KDE}$ employs the Fingerprinting data to estimate the target location.

\section{A. Fingerprinting}

Large variance of measurements gathered is one of the most critical problems associated to RSSI estimation, specially for indoor environments, even in case of static objects [20]. An example of this variance is shown in figure 1 where a large amount of RSSI measurements are taken from a static object equipped with several sensors. A common approach to deal with this issue is by applying Fingerprinting Technique [21], [22], [23]. This technique allows to obtain a proper scenario calibration by splitting it into a set of cells $\mathrm{c}=\left\{\mathbf{p}_{l} \mid l=\right.$ $1, \ldots, L\}$ with geometrical center at $\mathbf{p}_{l}=\left(x_{l}, y_{l}\right) \in \mathbb{R}^{2}$ respectively. The larger the number of cells $L$ is, the higher the spatial resolution is. Afterward, measures are taken from each node $v_{i}$ with the target object located at the center of every cell $\mathbf{p}_{l}$ for a predefined time interval $\tau$. The longer the calibration time is, the better the cell characterization is. The entire Fingerprint process is detailed in algorithm 1.

As a result of this procedure, a distribution of the RSSI is captured in every cell for every technology $\lambda$ by every node $v_{i}$. For this purpose, the multi-dimensional $\mathbf{F}$ matrix has been defined to contain the fingerprinting distributions. Therefore, $\mathbf{F}\left(\mathbf{p}_{l}, \lambda, \mathbf{R S S}\right)$ represents the set of fingerprint measurements gathered at cells position $\mathbf{p}_{l} \in \mathbb{R}^{2}$ by sensor technology $\lambda$ by nodes $v_{i} \in \mathbf{v}$. Moreover, $\mathbf{F}$ matrix is the input of the TPE process described in next subsection. As an example, in figure 2 are shown the fingerprint cells location for the experiments room presented in this work (left) and some examples of RSSI fingerprinting distributions (right).

\section{B. Target Position Estimation (TPE)}

There exist several works in the literature for position estimation based on Fingerprint statistics [39], [41], [42], [43]. Most of these works apply deterministic techniques 

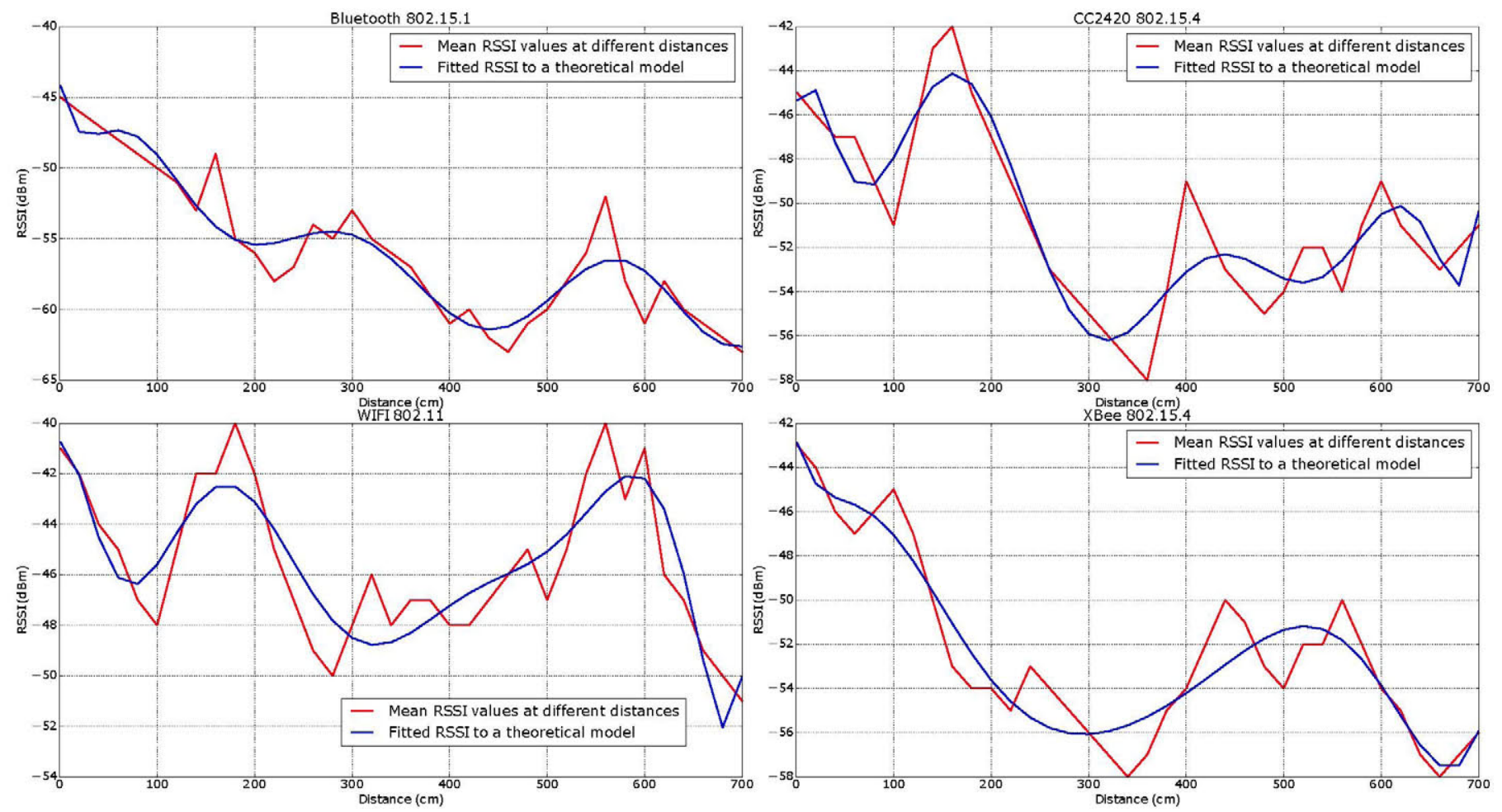

Fig. 1. RSSI behavior and variance along distance for different wireless sensors. IEEE802.11.g WiFi variation is lesser than other technologies due to the short-term attenuation for considered distances. Conversely, IEEE802.15.4 (XBee) example is easy to fit into a valid model due to its decreasing behavior.

to characterize the RSSI distribution for each cell. In [39], the K-Nearest Neighbors (KNN) technique is employed to triangulate the target position adopting euclidean distance as metric. In [41], a comparison of various metrics is carried out in an indoor building giving as result that Mahalanobis distance outperforms Manhattan and Euclidean-Distance results. Furthermore, in [42] a weighted K-Nearest Neighbor approach is presented. Moreover, in [43] it is proposed a complete overview of several deterministic methods that include all the aforementioned metrics. However, taking into consideration a large set of measures collected for every cell in Fingerprint stage, a deterministic approach can neglect the wide distribution of measurements per cell, therefore a probabilistic approach can be considered for the estimation.

Kernel Density Estimation (KDE) is a non-parametric technique employed to obtain the Probability Density Function (PDF from now on) of a random variable with independent and identically distributed samples [24], [25]. KDE estimates the likelihood that Fingerprinting distributions match the measures in the region of a Kernel function. Therefore, the distance is fitted with Kernel functions, denoted by $K(\cdot)$ and the PDF in our problem can be calculated by the following function:

$$
p\left(\mathbf{R S S}_{i, \lambda} \mid \mathbf{p}_{l}\right)=\frac{1}{N h_{N}} \sum_{n=1}^{N} K\left(\frac{R S S_{i, \lambda}-\mathbf{F}\left(\mathbf{p}_{l}, \lambda, \mathbf{R S S}_{n}^{i}\right)}{h}\right)
$$

where $N$ is the set of samples in every fingerprinting cell center $\mathbf{p}_{l}$ by the node $v_{i}$. Furthermore, $h$ is a smoothing parameter also known as kernel bandwidth. $R S S_{i, \lambda}$ is the node measurement. In addition, $\mathbf{F}\left(\mathbf{p}_{l}, \lambda, \mathbf{R S S}_{n}^{i}\right)$ is the set of
RSSI values gathered by node $v_{i}$ at fingerprint cell $\mathrm{p}_{l}$ with the corresponding technology $\lambda$.

The goal pursued is to estimate a non-parametric function that fits the RSSI values distribution better than known (parametric) distributions. Due to the nature of the data modeled, a modified version of KDE known as Nadaraya-Watson Kernel regression is used [26]. This method is applied because of its appropriateness when there is no prior knowledge of the relationship between the variables under study since these estimators are only based on either smoothing or regression functions.

There are other non-parametric options that could have been employed such as the Priestley-Chao and Gasser-Mller smoother or the K-th Nearest-Neighbor (K-NN) weights [40]. However, Priestly-Chao and Gasser-Mller strict boundary bias problems make Nadaraya-Watson method the most suitable estimator for RSSI-based applications. In fact, it has been demonstrated that Nadaraya-Watson variance is up to a $50 \%$ lesser than the other methods [40]. However, K-NN smoothing can attain similar results to the ones obtained by using kernel estimation when an appropriate bandwidth $h$ parameter is adjusted.

According to this method, the joint probability function can be estimated as:

$$
\begin{aligned}
p\left(\mathbf{p}, \mathbf{R S S}_{I, \lambda}\right)= & \\
& \frac{1}{L} \sum_{l=1}^{L} \frac{1}{\left(h_{\mathbf{x}}\right)^{2}\left(h_{N}\right)^{I}} K\left(\frac{d\left(\mathbf{p}, \mathbf{p}_{l}\right)}{h_{\mathbf{x}}}\right) \\
& K\left(\frac{\mathbf{R S S}_{I, \lambda}-\mathbb{E}\left[\mathbf{F}\left(\mathbf{p}_{l}, \lambda, \mathbf{R S S}\right)\right]}{h_{N}}\right)
\end{aligned}
$$



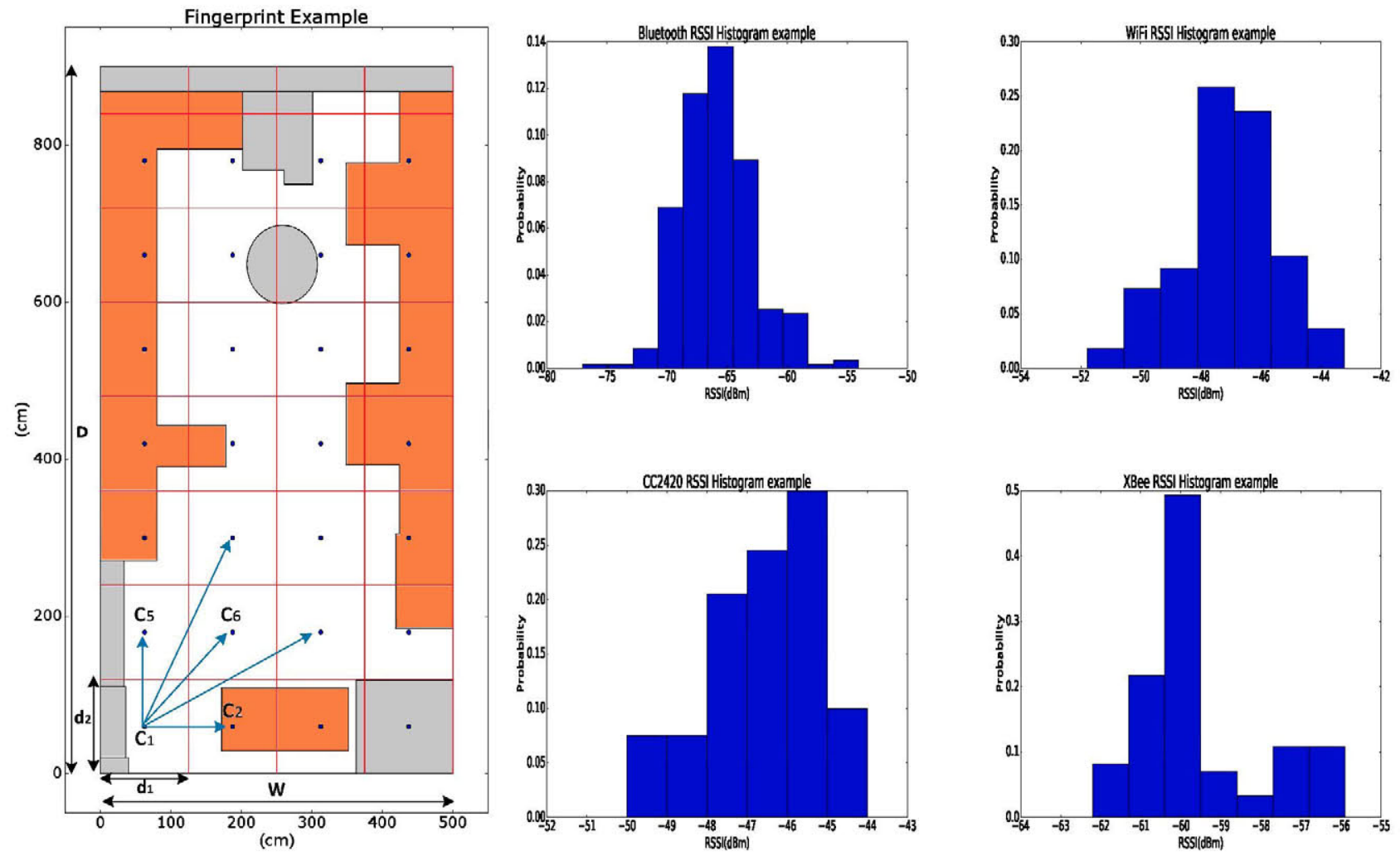

Fig. 2. (Left) Fingerprint cells distribution used in this work (Right). RSSI Histogram examples for each sensor in a particular cell. It can be observed that IEEE802.11.g WiFi arises narrow range of RSSI values for the cell considered whereas IEEE802.15.1 Bluetooth histogram probability distribution is wide spread.

where $d\left(\mathbf{p}, \mathbf{p}_{l}\right)$ represents the distance from every fingerprinting cell center to the estimated point and $h_{\mathbf{x}}, h_{N}$ are the bandwidth parameters respectively. Finally, the expectation $\mathbb{E}\left[\mathbf{F}\left(\mathbf{p}_{l}, \lambda, \mathbf{R S S}\right)\right]$ yields to a vector containing the RSS mean for every node.

Applying the Minimum Mean Square Error (MMSE) criteria over the conditional density probability obtained by Bayes theorem:

$$
\mathbf{z}=\hat{\mathbf{p}}_{M M S E}=E\left(\mathbf{p} \mid \mathbf{R S S}_{I, \lambda}\right)=\int_{C} \mathbf{p} p\left(\mathbf{p} \mid \mathbf{R S S}_{I, \lambda}\right) d x
$$

it is obtained that:

$$
\hat{\mathbf{p}}^{(\lambda)}=\sum_{l=1}^{L} w_{l} \mathbf{p}_{l}
$$

where $\mathbf{p}_{l}$ are the fingerprint cells center. Superscript is used to indicate that the positions estimated were obtained by using a particular technology. The target position $\hat{\mathbf{p}}$ is calculated as the weighted sum of the estimations for every cell using the Kernel function. The weights $w$ are determined by:

$$
w_{l}=\frac{K\left(\frac{\mathbf{R S S}_{I, \lambda}-\mathbb{E}\left[\mathbf{F}\left(\mathbf{p}_{l}, \lambda, \mathbf{R S S}\right)\right]}{h_{N}}\right)}{\sum_{j=1}^{L} K\left(\frac{\mathbf{R S S}_{I, \lambda}-\mathbb{E}\left[\mathbf{F}\left(\mathbf{p}_{j}, \lambda, \mathbf{R S S}\right)\right]}{h_{N}}\right)}
$$

The numerator contains the current Kernel for cell $l \in \mathbf{c}$ and the denominator collects the sum of all Kernel values for every cell. The Nadaraya-Watson Kernel regression is the MMSE estimator of $\mathbf{p}$. The TPE output is the estimated value $\mathbf{z}=\hat{\mathbf{p}}$ which is assumed to be static in $k$.

The parameter selection for the algorithm to fit the PDFs in equation (5) is carried out using the exponential kernel (6).

$$
K(\mathbf{x})=\frac{1}{2} \exp ^{(-\|\mathbf{x}\|)}
$$

where $\|\mathbf{x}\|$ represents the euclidean distance. There are several kernels in the literature but the computation complexity is higher. In our work, the last section VII presented that exponential kernel improve the estimation accuracy than using others most common kernels like the Gaussian kernel reducing the algorithm time.

Furthermore, the bandwidth selection is based on [48] where a study of different method are carry out to obtain this parameter. Based on the tables presented is this work the parameter is setting equal to $h_{N}=0.8$.

\section{TARgET TRACKING PROCESS (TTP)}

Tracking stage is intended to minimize the effect of noise in $\hat{\mathbf{p}}$ by filtering outliers giving as a result the peaks-free object route. In order to maintain notation in a simple manner, superscript used in equation (4) has been removed since the tracking system is described for a single technology. Several methods have been proposed for this purpose, however in 


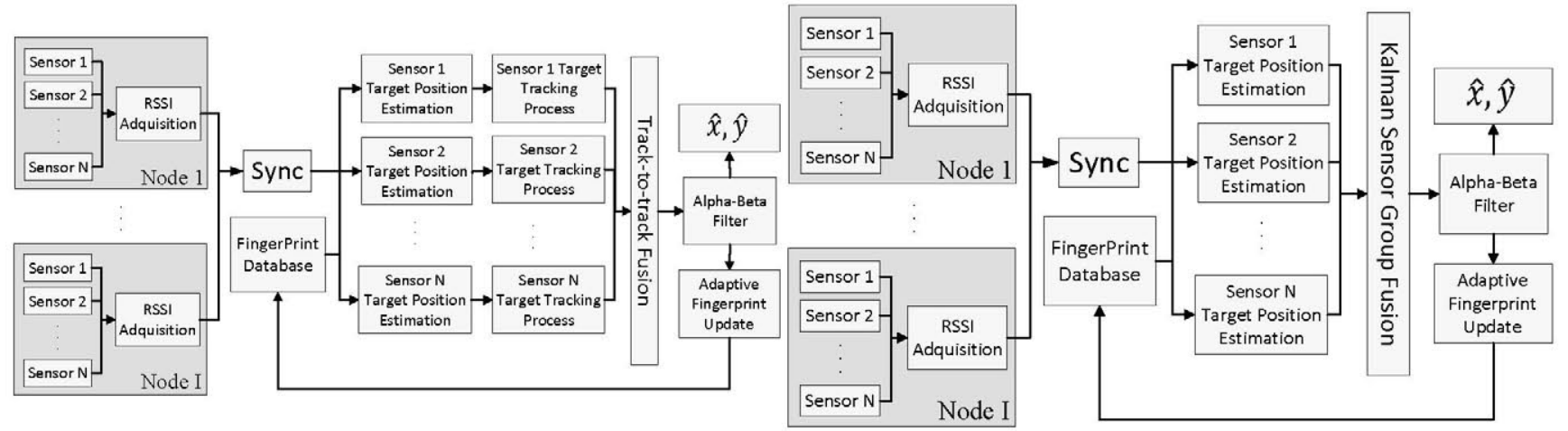

Fig. 3. Architecture patterns for sensor fusion. (left). Track-to-track Fusion: Separate processing stages for each sensor to achieve high-level inferences that are subsequently fused. (right) Kalman Sensor Group Fusion: direct sensor data combination.

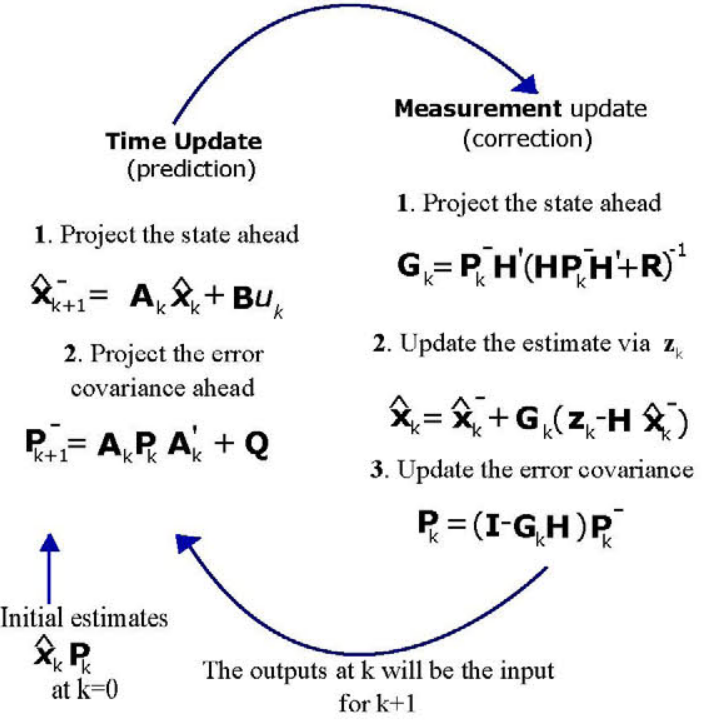

Fig. 4. Kalman Filter Algorithm steps for prediction and estimation.

this work the well-known Kalman Filter (KF) [27], [28] is employed. This filter provides a recursive solution based on Least Squares Method.

$\mathrm{KF}$ assumes the system to be described by a linear stochastic model, where the error associated to the system as well as the additional information incorporated are presented as normal distribution variables with zero mean and variance $\sigma^{2}$.

A complete illustration of Kalman Filter is drawn in figure (4). This filter performs the Best Linear Unbiased Estimator (B.L.U.E) of a system state using information from the previous estimations. KF consists of two stages: (a) time update (left) and $(b)$ measurement update (right) are related. The former is in charge of projecting future estimators of the current state and error covariance. The latter will predict new state estimations.

One of the most interesting KF features is the ability to predict the state of a system, even if the nature of the system modeled is unknown.

The Time Update (prediction) estimates the state $\mathbf{x}$ according to the following equation:

$$
\mathbf{x}_{k+1}=\mathbf{A}_{k} \mathbf{x}_{k}+\mathbf{B}_{k} u_{k}
$$

where A matrix relates the $k$ state with the $k+1$ time state. Furthermore, $\mathbf{B}$ is the control input associated to $u_{k}$, which is the system input. Furthermore, $\mathbf{P}$ matrix is the a posteriori error covariance matrix from Kalman process that collects the deviation in the estimations and $\mathrm{Q}$ is the covariance matrix of the process noise.

Moreover, Measurement update (correction) stage in figure 4 is described as follows:

(1): The matrix G, called Kalman Gain or blend factor, adjusts the trade-off between the estimation and the error measurement.

(2): Equation to calculate the posterior state $\mathbf{x}_{k+1}$ as a linear combination of the a prior $\mathbf{x}_{k}$ estimator and the weighted difference between current observations $\mathbf{z}_{k}$ and a measurement prediction $\mathbf{H}$.

(3): Update the error covariance based on the Kalman Gain. Moreover, the observation $\mathbf{z} \in \mathbb{R}^{2}$ contains the spatial coordinates of the position :

$$
\mathbf{z}_{k}=\mathbf{H} \mathbf{x}_{k}+\eta_{k}
$$

Finally, matrix $\mathbf{H}$ relates the state with the measure $\mathbf{z}_{k}$ where $\mathbf{z}_{k}$ is the TPE output and $\eta \sim \mathcal{N}(0, \sigma)$ the measurement noise respectively.

In this paper, a Modified version of the Kalman Filter (MKF) [31] is applied. The reason to use MKF is that the model considered in this system is linear. Consequently, a linear version of $\mathrm{KF}$ taking into consideration velocity and acceleration parameters represents a good trade-off between complexity (i.e Extended Kalman Filter) and performance (velocity and acceleration improve the estimation results). In addition, Extended Kalman Filter does not provide the optimal solution in case of wrong initial state inputs, which is highly probable in the scenarios considered in this work [44].

Therefore, MKF algorithm considers the speed axis $v_{x}$ and $v_{y}$ variables for the state $\mathrm{x}$ in addition to the position vector $\hat{\mathbf{p}} \rightarrow(\hat{x}, \hat{y})$ of the tracked target. These variables in combination with the use of a more realistic movement model known as Random Walk Model [49] represent an advantage in terms of estimation accuracy. 
Therefore, the system can be expressed as follows:

$$
\mathbf{A}_{k}=\left[\begin{array}{cccc}
1 & 0 & d t & 0 \\
0 & 1 & 0 & d t \\
0 & 0 & 1 & 0 \\
0 & 0 & 0 & 1
\end{array}\right]
$$

where the states $\mathbf{x}_{k}$ are composed by axis positions and speed:

$$
\mathbf{x}_{k}=\left[x, y, v_{x}, v_{y}\right]^{\prime}
$$

Additionally, $\mathbf{H}$ remains as the general Kalman formulation whereas $\mathbf{B}_{k}$ will be modified as follows:

$$
\mathbf{H}=\left[\begin{array}{cccc}
1 & 0 & 0 & 0 \\
0 & 1 & 0 & 0
\end{array}\right], \quad \mathbf{B}_{k}=\left[\begin{array}{c}
\frac{1}{2} d t^{2} \\
\frac{1}{2} d t^{2} \\
d t \\
d t
\end{array}\right]
$$

Main modifications presenting the movement information and are included in different matrices of the process, where $u_{k} \equiv \sigma_{a}^{2}$ is the acceleration parameter. This parameter is set to 0.1 based on the normal people velocity when they are walking. Moreover, $\mathbf{R}$ matrix contains the error variance of TPE estimation:

$$
\mathbf{R}=\left[\begin{array}{cc}
\sigma_{x}^{2} & 0 \\
0 & \sigma_{y}^{2}
\end{array}\right]
$$

where $\sigma_{x}, \sigma_{y}$ represent the error deviation in TPE estimation in $x$ and $y$ respectively and are settled to the TPE mean error for each technology used. The values of $\mathbf{R}$ were obtained by performing several independent experiments for each of the technologies considered in this work. Note that consequently, the Kalman Filter has its own $\mathbf{R}$ Estimation Error Covariance Matrix corresponding to IEEE802.15.1, IEEE802.15.4 and IEEE $802.11 \mathrm{~g}$ standards respectively.

Finally, according to the Random Walk Model, the covariance matrix $\mathbf{Q}$ :

$$
\mathbf{Q}=\left[\begin{array}{cccc}
\frac{1}{4} d t^{4} & 0 & \frac{1}{2} d t^{3} & 0 \\
0 & \frac{1}{4} d t^{4} & 0 & \frac{1}{2} d t^{3} \\
\frac{1}{2} d t^{3} & 0 & d t^{2} & 0 \\
0 & \frac{1}{2} d t^{3} & 0 & d t^{2}
\end{array}\right] \sigma_{q}^{2}
$$

where $\sigma_{q}$ parameter must be fixed. For people acceleration in a normal walking behavior, it is equal to 1 . This value has been chosen according to the behavior estimation proposed in [29], [30].

Finally, for the sake of simplicity, it is assumed that the outputs of the KF are denoted using the same notation than previous sections. Therefore, the outputs are $\hat{\mathbf{p}}_{k}=\hat{x}_{k}, \hat{y}_{k}$ for estimated positions and $\mathbf{P}_{k}$ for error covariance.

\section{Multi-SEnsor Processing ARCHitectures}

In this paper, mainly two multi-sensor schemes are considered: (1) Track-To-Track Fusion (TTTF) and (2) Kalman Sensor Group Fusion Architecture (KSGF). Both architecture patterns are shown in figure 3 . In the former, processing stages (TPE and TTP) are performed separately for each sensor to achieve high-level inferences (routes) that are subsequently fused. Nonetheless, in the latter scheme the sensor measurements are combined in the TTP stage. Both schemes are described and compared in next subsections and results are presented in section VII.

\section{A. Track-To-Track Fusion Architecture TTTF}

This architecture pattern relies on fusing the routes obtained by each technology $\lambda$ separately. The proposed algorithm applies the Maximum Likelihood Estimator (MLE) to the probability density function of the available routes [32]. For this purpose, $\Re$ is defined to be the set of routes. Additionally, it is assumed that every route is composed by $\varpi$ number of steps $\mathfrak{R}^{\lambda}=\left\{\hat{\mathbf{p}}_{1}, \ldots, \hat{\mathbf{p}}_{\varpi}\right\}$. Notice that the algorithm is able to fuse information even if there is not measurements from all technologies available in the time window considered. Therefore, the probability density function can be described as follows:

$$
p\left(\mathfrak{R}^{\Lambda}\right)=\prod_{k=1}^{\varpi} \prod_{\lambda=1}^{\Lambda} \frac{1}{\sqrt{(2 \pi)^{\mathfrak{D}}\left|\mathbf{P}_{\lambda, k}\right|}} e^{-\frac{1}{2}\left(\hat{\mathbf{p}}_{k}-\overline{\mathbf{p}}_{k}\right)^{\prime} \mathbf{P}_{\lambda, k}^{-1}\left(\hat{\mathbf{p}}_{k}-\overline{\mathbf{p}}_{k}\right)}
$$

where $\mathbf{P}_{\lambda, k}$ and $\mathbf{p}_{k}$ are the outputs from TTP described in section IV. Note that $\hat{\mathbf{p}}$ describes the position estimated from previous stages (TPE and TTP), whereas $\overline{\mathbf{p}}$ denotes the final output of the Fusion stage. Moreover, $\mathfrak{d}$ is the range of $\mathbf{P}$, which denotes the number of sensors available in a particular window-time in the network. From equation (14), it has been demonstrated [45] that MLE can be obtained by minimizing the result of $\overline{\mathbf{p}}$ and setting the gradient equal to 0 . As a result, the state estimation for every location is calculated as follows:

$$
\overline{\mathbf{p}}_{k}=\left(\sum_{\lambda=1}^{\Lambda} \mathbf{P}_{\lambda, k}^{-1}\right)^{-1} \sum_{\lambda=1}^{\Lambda} \mathbf{P}_{\lambda, k}^{-1} \hat{\mathbf{p}}_{k}
$$

Note that, both the estimation $\overline{\mathbf{p}}_{k}$ and $\mathbf{E}\left(\overline{\mathbf{p}}_{k}\right)=\overline{\mathbf{p}}_{k}$ are unbiased. Finally, the covariance of the estimate, denoted as $\Sigma$, is given by:

$$
\Sigma_{k}=\left(\sum_{\lambda=1}^{\Lambda} \mathbf{P}_{\lambda, k}^{-1}\right)^{-1}
$$

It can be shown that this result is the Kalman Filter update equation applied to each local route in each partition. Further details can be found in [45].

\section{B. Kalman Sensor Group Fusion Arquitecture KSGF}

The second architecture presented is the Kalman Sensor Group Fusion (KSGF) which exploits the Kalman filter properties for multi-sensor data fusion [33]. KSGF method directly incorporates the position estimations of each sensor in a single Kalman Filter. However, the system must be properly conditioned to obtain an accurate route estimation.

General KF system equations were described in section IV. However, some modifications must be introduced in the filter to support multi-sensor functionalities. In concrete, update matrix $\mathbf{H}$, described in equation (11), must be adapted to incorporate the sensor inputs: 


$$
\mathbf{H}=\left[\begin{array}{llll}
1 & 0 & 0 & 0 \\
0 & 1 & 0 & 0 \\
1 & 0 & 0 & 0 \\
0 & 1 & 0 & 0 \\
\dot{1} & \cdot & \cdot & 0 \\
1 & 0 & 0 & 0 \\
0 & 1 & 0 & 0
\end{array}\right]
$$

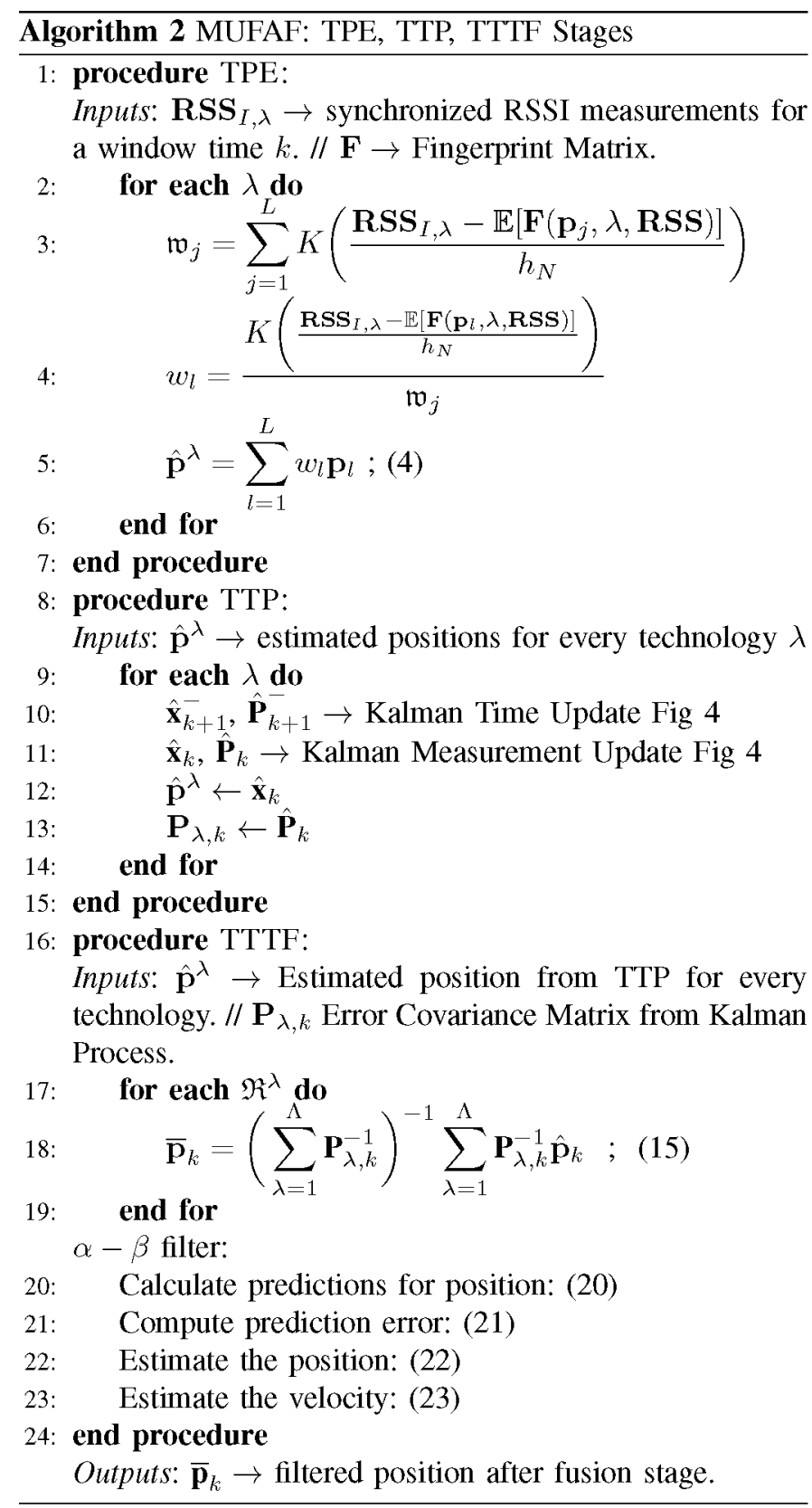

Furthermore, $\mathbf{R}$ matrix must include sensor error covariance measurements. These values are appended to the main diagonal:

$$
\mathbf{R}=\left[\begin{array}{cccccccc}
\sigma_{x_{1}}^{2} & 0 & 0 & 0 & 0 & 0 & 0 & 0 \\
0 & \sigma_{y_{1}}^{2} & 0 & 0 & 0 & 0 & 0 & 0 \\
0 & 0 & \sigma_{x_{2}}^{2} & 0 & 0 & 0 & 0 & 0 \\
0 & 0 & 0 & \sigma_{y_{2}}^{2} & 0 & 0 & 0 & 0 \\
. & . & . & . & . & . & . & . \\
0 & 0 & 0 & 0 & 0 & 0 & \sigma_{x_{\Lambda}}^{2} & 0 \\
0 & 0 & 0 & 0 & 0 & 0 & 0 & \sigma_{y_{\Lambda}}^{2}
\end{array}\right]
$$

where $\sigma$ parameters are the same that have been used in the TTP process for each independent technology.

Finally, the observations vector $\mathbf{z}$ is now extended to collect the $x$ and $y$ coordinates estimated from each sensor:

$$
\mathbf{z}=\left[\begin{array}{lllllll}
\hat{x}_{1}, & \hat{y}_{1}, & \hat{x}_{2}, & \hat{y}_{2}, & \ldots & \hat{x}_{\Lambda}, & \hat{y}_{\Lambda}
\end{array}\right]^{\prime}
$$

Taking into consideration the aforementioned modifications, the KF can be performed as described in TTP. As a result, the fused route will be provided by the KSGF. Both architecture patterns can be employed for multi-sensor data fusion. The computational complexity of the architectures proposed has been addressed [34] and detailed in section VII. In this work, both patterns are compared in terms of execution time. KSGF computing time grows exponentially as the number of sensors increases.

\section{Alpha-Beta Filter for noise reduction}

Fusion stage improves the quality of the estimated route in general terms as it takes the information from all sensor technologies. However, due to the high values in covariance matrices employed for Multi-Sensor fusion stage, some outliers can arise or propagate. Additionally, as the Multi-Sensor Fusion can calculate routes with partial information (even a single technology with a high error rate), the outliers can appear or introduce noise in future estimations. These outliers corrupt the normal target path which can yield to inappropriate routes modeling or wrong patterns generation among others.

In order to minimize the noise and outliers effect that can arise as result of multi-sensor stage, an additional filtering stage is applied. There exist several proposals for routes smoothing, however in this paper, the well known Alpha-Beta filter [35], [36] is used. The reasons to employ the Alpha-Beta filter are the lower complexity, the lesser computational cost and that system model details are not required. Furthermore, taking into consideration that the effect of outliers is much lesser in the presence of low noisy estimations, it has been shown that the alpha-beta filter estimations performance is similar to Kalman Filters [47].

Similarly to equation (9), initial states of spatial coordinates and axis speeds are considered. From Fusion stage, the notation for estimated positions is $\overline{\mathbf{p}}=\left(x_{m}, y_{m}\right)$. This position is the input of the Alpha-Beta filter. Therefore, the position prediction can be obtained as follows:

$$
\hat{x}_{k+1}=\hat{x}_{k}+\hat{v}_{x_{k}} d t ; \hat{y}_{k+1}=\hat{y}_{k}+\hat{v}_{y_{k}} d t
$$

Additionally, error measurements can be calculated from predicted states by the subtraction of the fusion algorithm $\hat{x}_{m}, \hat{y}_{m}$ from the predicted position: 


$$
e_{x}=\hat{x}_{m}-\hat{x}_{k+1} ; e_{y}=\hat{y}_{m}-\hat{y}_{k+1}
$$

The final position and velocity for next algorithm iterations are obtained as follows:

$$
\begin{gathered}
\hat{x}_{k}=\hat{x}_{k+1}+\alpha e_{x} ; \hat{y}_{k}=\hat{y}_{k+1}+\alpha e_{y} \\
\hat{v}_{x_{k}}=\hat{v}_{x_{k}}+\frac{\beta}{\Delta t} e_{x} ; \hat{v}_{y_{k}}=\hat{v}_{y_{k}}+\frac{\beta}{\Delta t} e_{y}
\end{gathered}
$$

where $\alpha$ and $\beta$ are the filter control parameters. In order to guarantee filter stability and convergence, these values must fulfill the following conditions:

$$
0<\alpha<1 ; 0<\beta \leq 2 ; 0<4-2 \alpha-\beta
$$

Additionally, there is a direct relation between alpha-beta control parameters:

$$
\beta=\frac{\alpha^{2}}{2-\alpha}
$$

The $\alpha$ value is commonly settled to small values $(\leq 0.3)$ in order to attain a high smoothing filtering able to minimize the outliers impact in the final estimated path.

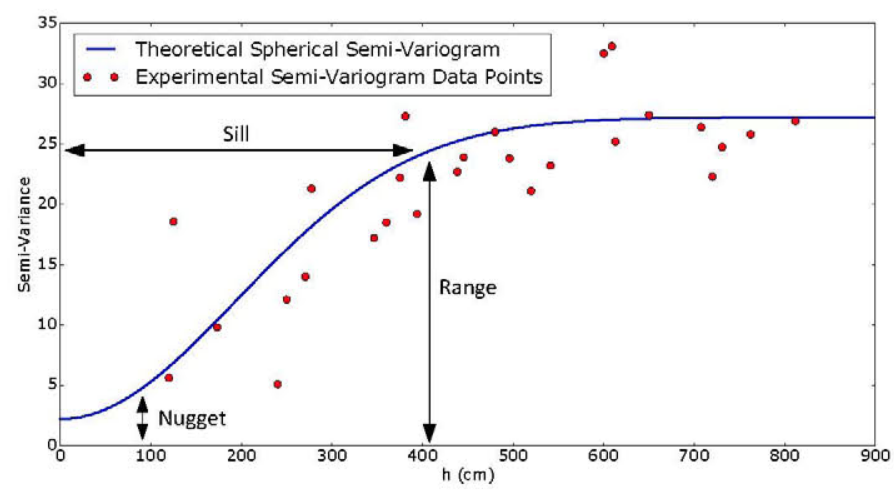

Fig. 5. Main Semivariogram Patterns: Nugget effect: uncertainty due to lack of very close samples. Range: larger distances than Range are uncorrelated. Sill: maximum similarity value.

The entire procedure for MUFAF Multi-Sensor Fusion is described in algorithm 2. In this pseudo-code the sequences to perform the TPE, TTP and TTTF stages are shown for an iteration.

\section{ADAPTIVE FINGERPRINTING UPDATE}

In this section, the Adaptive Fingerprint Update (AFU) stage is described. Wireless channel propagation is a very tough behavior to be mathematically modeled. Even the probabilistic methods lack of an accurate characterization due to several factors such as obstacles, reflection and multipath. Additionally, these undesired events are time-varying which makes it even harder to deal with. As described in section III, Fingerprinting technique face this problem by building a dataset to compare the correlation degree in indoor areas with statistical functions. However, this dataset must change if the propagation conditions vary. Time analysis can be applied to make predictions on the evolution of this channel [21].

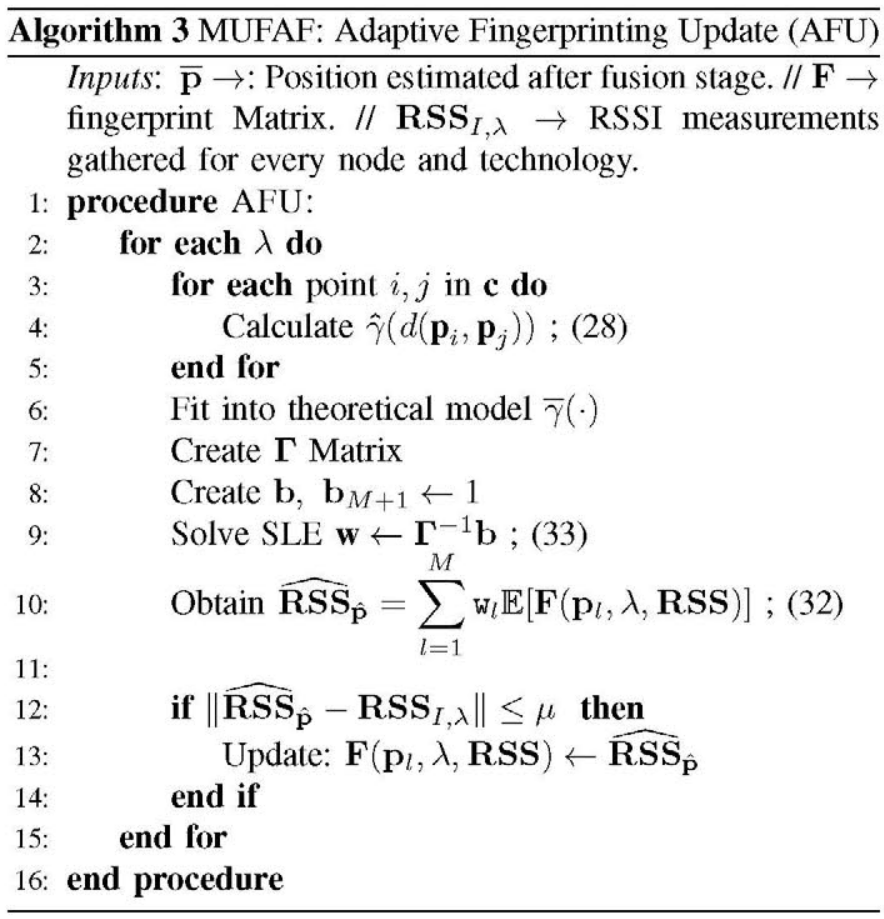

However, an interesting approach is reached by adaptively update the fingerprinting dataset based on the quality of the information gathered. Consequently, a proposal for AFU is presented in this work. This algorithm endows the entire MUFAF framework with responsiveness to indoor condition changes.

AFU can be described as follows: the input of the system is the RSSI values from the target tracked and the outputs from TPE, TTP and Fusion stages are the estimated positions of the mentioned target. AFU aim is to verify the similarity degree of the RSSI gathered with the value estimated at this location using the Fingerprint dataset. There exist a wide range of classical estimators in the literature. However, an interesting approach is given by the use an interpolation technique called Kriging [36], [37].

This technique was initially employed for geospatial analysis, however the method has been successfully applied to several sciences. The main goal of Kriging is to estimate the field behavior at unknown locations based on the available measurements.

In general terms, Kriging is a Best Linear Unbiased Estimator (B.L.U.E) which aims to minimize the mean square error. As aforementioned, if $\mathrm{z}$ denotes the observation:

$$
\operatorname{minimize} \quad[\hat{\mathbf{z}}(\hat{\mathbf{p}})-\mathbf{z}(\hat{\mathbf{p}})]
$$

The main feature (and differential) of Kriging interpolation is that guarantees to be unbiased even if stationary of field is not known. This can be expressed as:

$$
\mathbb{E}[\hat{\mathbf{z}}(\hat{\mathbf{p}})-\mathbf{z}(\hat{\mathbf{p}})]=0
$$

Statement (27) represents the main advantage over traditional methods based on covariance. This condition is satisfied due to the use of a statistical tool called semivariance. Its 
general estimator is given by the Experimental Variogram, denoted by $\hat{\gamma}(\cdot)$ :

$$
\hat{\gamma}(h)=\frac{1}{2 N(h)} \sum_{i, j=1}^{N(h)}\left(\mathbf{z}\left(\mathbf{p}_{i}\right)-\mathbf{z}\left(\mathbf{p}_{j}\right)\right)^{2}
$$

where $N(h)$ represents the number of RSSI measurements gathered at a predefined distance $h$.

The Semivariance is employed to exploit the spatial similarity without dependency of the field mean. Additionally, square difference (28) endows the estimator with robustness to field outliers.

Specifically, in MUFAF all fingerprint cells $\mathbf{c}$ are employed to build the Semivariogram:

$$
\hat{\gamma}_{\lambda}(\mathbf{h})=\frac{1}{2\|\mathbf{c}(\mathbf{h})\|} \sum_{\mathbf{c}(\mathbf{h})}\left(R S S_{\mathbf{p}_{i} \lambda}-R S S_{\mathbf{p}_{j}, \lambda}\right)^{2}
$$

$\forall i, j \in \mathbf{c} \wedge d\left(\mathbf{x}_{i}, \mathbf{x}_{j}\right) \in \mathbf{h}$. This process is performed $\forall v_{i} \in \mathbf{v}$. In the case of square/rectangular grids as the ones considered in figure 2, the lags distribution can be expressed as:

$$
d\left(x_{i}, y_{j}\right)=\sqrt[2]{\left(\frac{d_{1}}{2} x_{i}-\frac{d_{2}}{2} x_{j}\right)^{2}+\left(\frac{d_{1}}{2} y_{i}-\frac{d_{2}}{2} y_{j}\right)^{2}}
$$

where $x_{i}, y_{j} \in \mathbf{c}$ are the spatial coordinates of the cells center. Furthermore, let assume that $W$ and $D$ are the indoor width and depth expressed in centimeters respectively. Additionally, $L=\mathfrak{n} \times \mathfrak{m}$ is the total number of cells and $\mathfrak{n}$ and $\mathfrak{m}$ are the number of cells per side (width and depth). Finally, $d_{1}, d_{2}$ denote the distance from the center of a cell to the center of a neighbor cell in every axis. Consequently, it has been shown [46] that if the number of intervals within lags in $\mathbf{h}$ satisfies to be lesser than $\sqrt[2]{\frac{\pi \sqrt{\sqrt{W^{2}+D^{2}}}}{\log \left(\sqrt{\left.W^{2}+D^{2}\right)}\right.}}$ then it will exist at least a Semivariance measurement in every $\mathbf{h}$ interval.

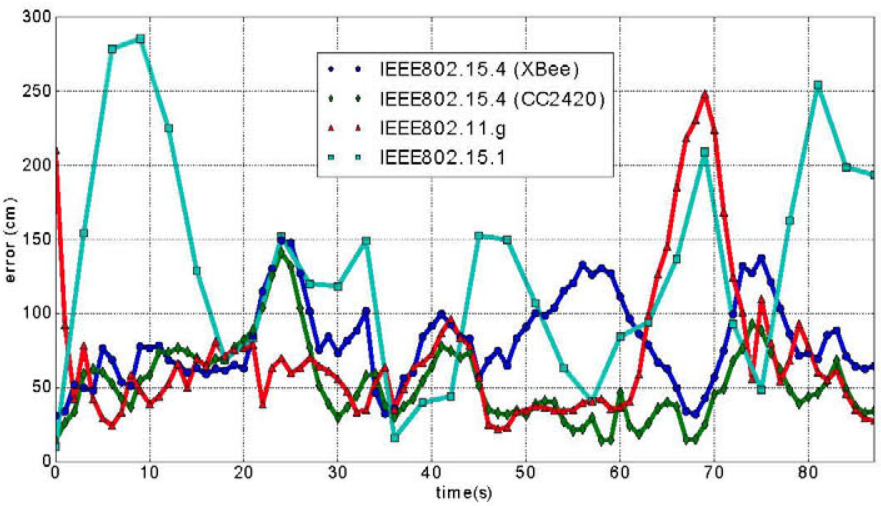

Fig. 6. Average error comparison of route estimation for every technology (no fusion applied) using Fingerprinting technique, Kernel Density Estimation and Kalman Filter.

Once the Experimental Semivariogram is calculated, a fitting into a general theoretical model, denoted by $\bar{\gamma}(\cdot)$ is required to infer spatial data for distances not considered in $\hat{\gamma}(\cdot)$.
There exist several theoretical models for the Semivariogram such as Linear, Spherical, Gaussian and Exponential [38]. Gaussian model is appropriated for processes with small short-term variations of similarity whereas Spherical or Exponential are the most suitable options for applications where considerable variations arise in short-distance. Due to the high variance in measurements distribution presented in section II, in this paper, Spherical Semivariogram theoretical model is employed:

$$
\bar{\gamma}(h)=n g+R g\left(\frac{3 h}{2 S i}-\frac{h^{3}}{2 S i^{3}}\right)
$$

where $n g$ is called the nugget effect. This parameter considers the uncertainty of the Semivariogram estimation when the separation between points tends to $0 . R g$ is the Range, that is the maximum distance taken into consideration for two samples. Finally, the Sill (Si) is the maximum variance (dissimilarity) between two samples. These parameters are drawn in figure 5.

As aforementioned, Kriging provides the (B.L.U.E). Therefore, the field value $\widehat{\mathbf{R S S}}_{\hat{\mathrm{p}}}$ can be described as a weighted sum of the available node measurements:

$$
\widehat{\operatorname{RSS}}_{\hat{\mathbf{p}}}=\sum_{l=1}^{M} \mathrm{w}_{l} \mathbb{E}\left[\mathbf{F}\left(\mathbf{p}_{l}, \lambda, \mathbf{R S S}\right)\right]
$$

where $\mathrm{w}=\left\{\mathrm{w}_{1}, \ldots, \mathrm{w}_{M}\right\}$ are the weights which sum must fulfill $\sum \mathrm{w}=1$ to guarantee unbiasedness. Furthermore, the weights are obtained by solving the following System of Linear Equations (SLE):

$$
\Gamma \mathbf{s}=\mathbf{b}
$$

where:

$$
\boldsymbol{\Gamma} \equiv \begin{cases}\bar{\gamma}\left(d\left(\mathbf{p}_{i}, \mathbf{p}_{j}\right)\right), & i, j=1, \ldots, M \\ 1, & i=M+1, j=1, \ldots, M \\ & j=M+1, i=1, \ldots, M \\ 0, & i, j=M+1\end{cases}
$$

is an $M+1 \times M+1$ Matrix capturing the spatial similarity among the available nodes in the fingerprint cell distribution. Additionally:

$$
\mathrm{s}=[\mathrm{w}, \mathcal{L}]^{\prime}
$$

s vector contains the weights w and the $M+1$ value is the Lagrange Multiplier $\mathcal{L}$. that ensures the unbiased nature of the estimator and

$$
\mathbf{b}=\left[\bar{\gamma}\left(d\left(\hat{\mathbf{p}}, \mathbf{p}_{1}\right)\right), \bar{\gamma}\left(d\left(\hat{\mathbf{p}}, \mathbf{p}_{2}\right)\right), \ldots, \bar{\gamma}\left(d\left(\hat{\mathbf{p}}, \mathbf{p}_{M}\right)\right), 1\right]^{\prime}
$$

b vector contains set of theoretical semivariances from the nodes to the estimated point $\hat{\mathbf{p}}$.

Finally, by solving the aforementioned System of Linear Equations SLE (33), the RSSI estimation is obtained. However, to determine the fingerprint update, the following decision rule has been implemented:

$$
\left\|\widehat{\mathbf{R S S}}_{\hat{\mathbf{P}}}-\mathbf{R S S}_{I, \lambda}\right\| \leq \mu
$$



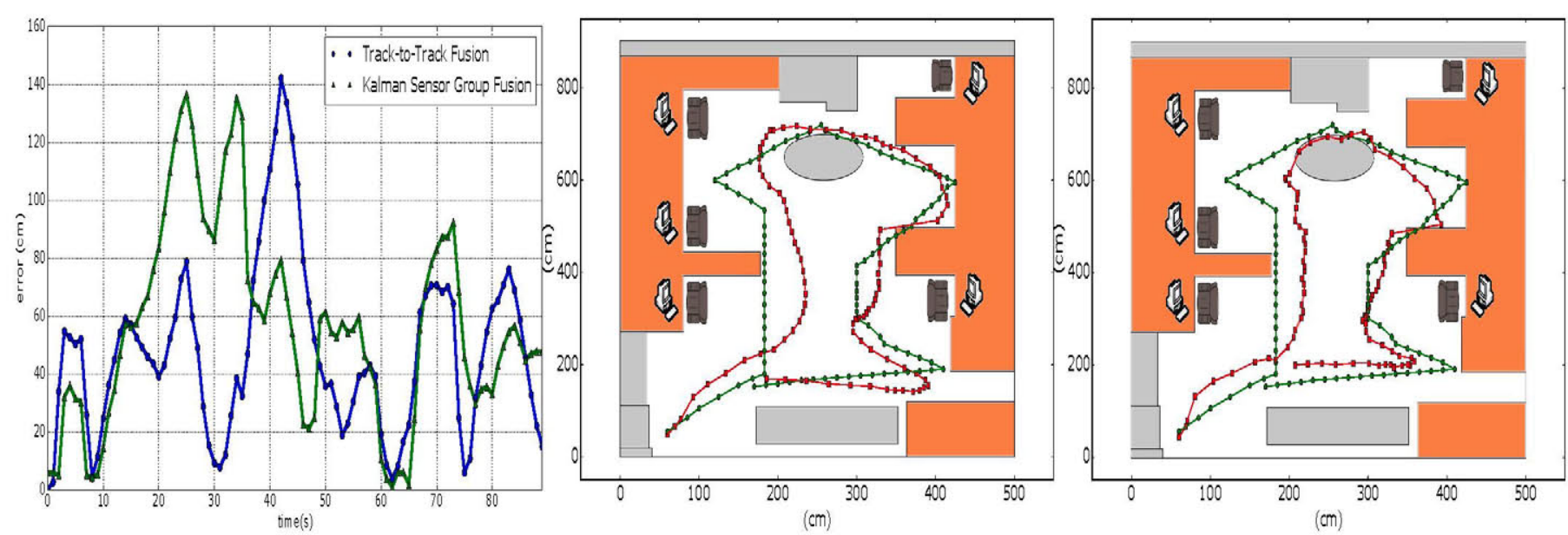

Fig. 7. (Left) Average error using both fusion architectures in path estimation for an experiment for a particular experiment with 90 steps per route. (Center) Path estimation by using Track-To-Track Fusion Architecture and (Right) Path estimation by using Kalman Sensor Group Fusion Architecture where real person route is denoted by green dotted line and MUFAF Route reconstruction is represented by red dotted line.

where $\mu$ is an empirical threshold which value must be iteratively calculated. The process consists on: $(a)$ perform a significant number of randomly generated routes (i.e for the experiments carried out 1000 routes were generated). (b) For these routes, the decision threshold must be initially adjusted to a low value (i.e $\mu=10^{-1}$ ) which implies a low update rate as most of the measurements will be neglected in the fingerprint dataset. (c) Calculate the mean error of the routes with this threshold. (d) Iteratively increase the $\mu$ value until a large number (i.e the high boundary in experiments presented in this work was settled to 5) which means that all measurements will be accepted to update the fingerprint dataset and repeat the mean error calculation. (e) Find the $\mu$ value that minimizes the mean error function.

The general outline for Kriging implementation is described in algorithm 3. In this algorithm, the sequential steps to obtain the RSSI estimation and to compare it with data gathered (RSSI from sensors) in order to make a decision on the Fingerprint update stage.

In addition to the theoretical model choice for the Semivariogram, there are some parameters that can affect the proper functionality of the Kriging interpolation. A critical parameter is the decision threshold $\mu$. The process aforementioned needs to be perform with no prior knowledge of the network behavior. However, the better the process is carried out the higher the precision and responsiveness of MUFAF algorithm will be. Moreover, the number of lags $(\mathbf{h})$ is relevant to attain an appropriate fitting. Furthermore, the number of neighbors is important to reach the best trade-off between complexity and accuracy. In next section, experiments executed as well as the results obtained will allow a better understanding of the process described in this section as well as the improvement of the results compared with classical estimation techniques.

\section{EXPERIMENTAL RESULTS}

\section{A. Experiment Setup}

In this section, the experiments performed to assess MUFAF and the results obtained in comparison with existing methods are detailed. In the simulations, the laboratory scenario drawn

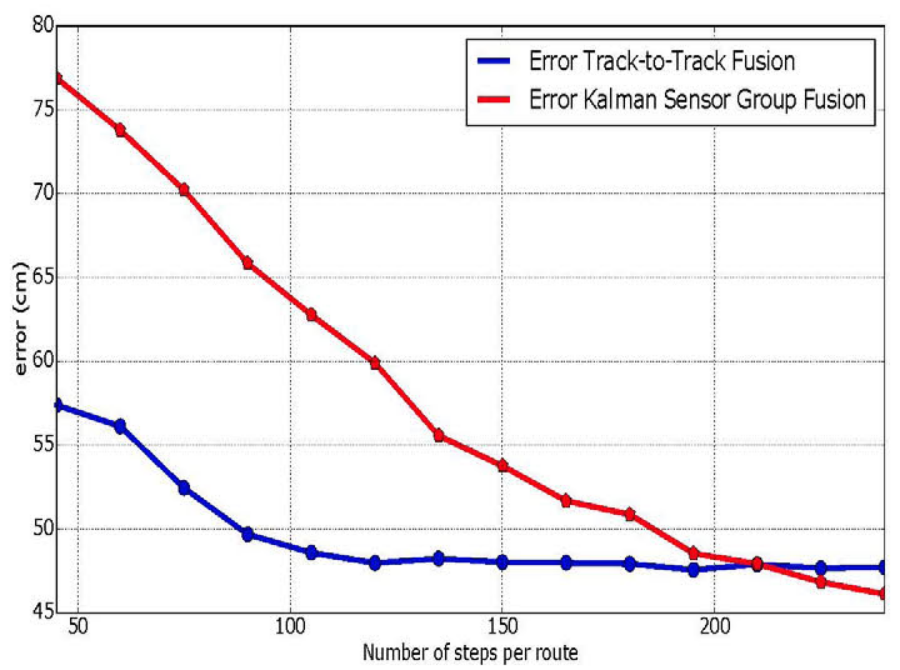

Fig. 8. Estimation error comparison for both architectures for several estimation periods. This experiment was performed using 1000 routes. KSGF error is lesser than TTTF in long term evolution.

in figure 2 is considered. This room represents a typical indoor environment with human activity, people flow controlled and harsh propagation conditions due to multipath fading, obstacles due to furnitures and wireless interferences due to electronic devices, further details can be found in [18].

Additionally, as described in section II, four $\mathbf{v}=v_{1}, \ldots, v_{4}$ nodes are deployed at the lab corners. Furthermore, every node is equipped with four sensors $\Lambda=4$ : IEEE802.15.1 (Bluetooth), IEEE802.11.g (WiFi), and two IEEE802.15.4 receivers (XBee and CC2420). Firstly, TPE and TPP performance are assessed by comparing MUFAF to other methods that employ fingerprint technique. Afterward, both pattern architectures are compared to observe their accuracy expressed in centimeters. Subsequently, experiments of Kriging interpolation are contrasted with covariance-based methods. Moreover, a complete framework experiment is carried out for multi-person tracking. Several aspects such as the number of steps per route, the number of neighbors available and the time-evolution of AFU 

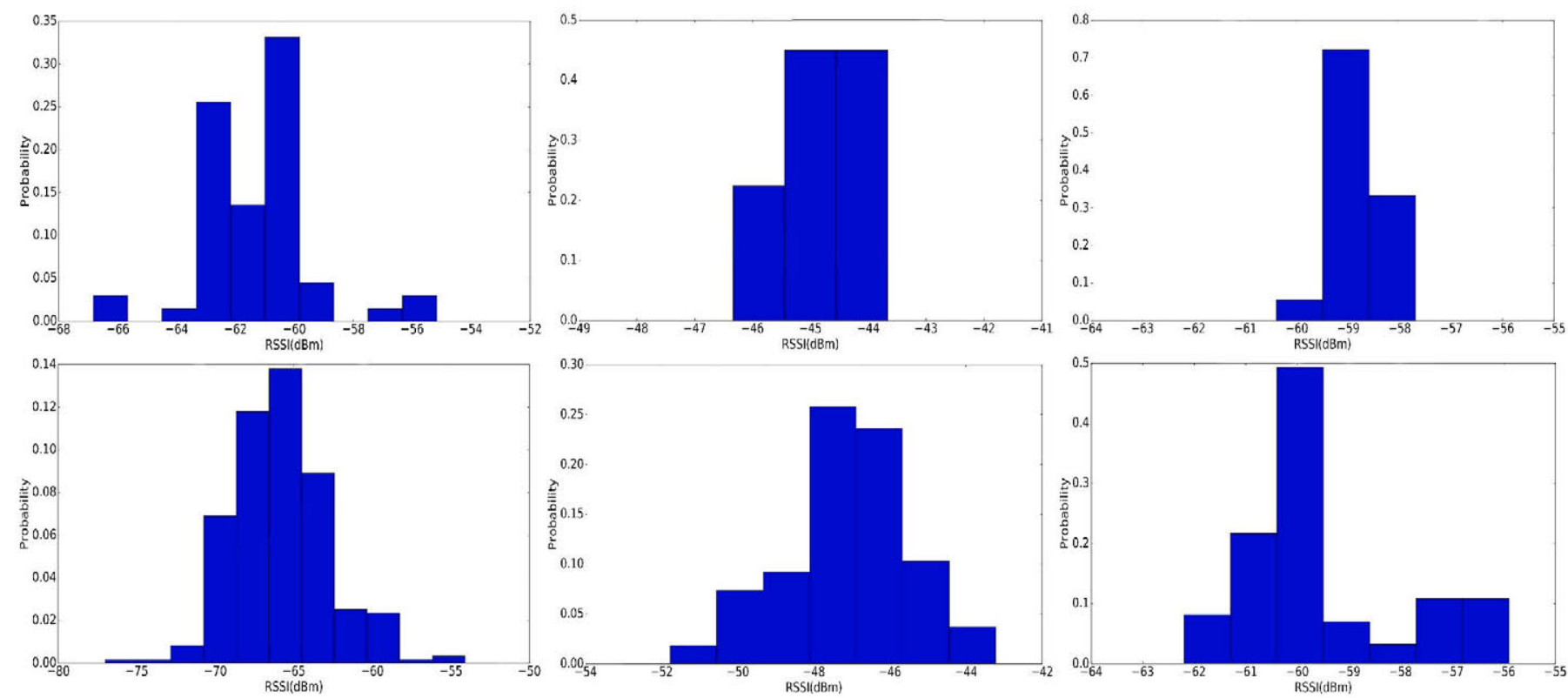

Fig. 9. Top graphics represent the initial histogram representation for a cell of 50 measurements whereas bottom shows the evolution of Histograms in 1000 simulations provided by AFU. (Left) Evolution of Histogram for AFU in IEEE802.15.1Bluetooth. (Center). Evolution of Histogram for AFU in IEEE802.11.g WiFi. (Right) Evolution of Histogram for AFU in 802.15.4.

TABLE II

AVERAGE ERROR COMPARISON OF MUFAF WITH SEVERAL METHODS EXPRESSED IN CENTIMETERS.

\begin{tabular}{|c|c|c|c|c|}
\hline & $\begin{array}{c}\text { IEEE } \\
802.15 .4 \\
\text { (XBee) }\end{array}$ & $\begin{array}{c}\text { IEEE } \\
(\mathrm{CC} 242.15 .4\end{array}$ & $\begin{array}{c}\text { IEEE } \\
802.11 . \mathrm{g} \\
\text { WiFi }\end{array}$ & $\begin{array}{c}\text { IEEE } \\
802.15 .1 \\
\text { Bluetooth }\end{array}$ \\
\hline $\begin{array}{c}\text { Euclidean } \\
\text { Distance } \\
{[41]}\end{array}$ & 110.71 & 150.367 & 122.72 & 150.57 \\
\hline $\begin{array}{c}\text { Mahalanobis } \\
\text { Distance } \\
{[41]}\end{array}$ & 108.33 & 140.05 & 112.12 & 142.58 \\
\hline K-NN [42] & 105.87 & 139.58 & 157.42 & 161.29 \\
\hline $\begin{array}{c}\text { Gaussian } \\
\text { Kernel [39] }\end{array}$ & 67.27 & 61.92 & 63.30 & 170.34 \\
\hline $\begin{array}{c}\text { MUFAF } \\
\text { Exponential } \\
\text { Kernel }\end{array}$ & 63.05 & 59.49 & 79.73 & 137.15 \\
\hline
\end{tabular}

were assessed in the results presented.

\section{B. Computational Cost}

The entire process for MUFAF implementation can be stated as:

- Create Fingerprint dataset.

- Performing TPE.

- Performing TPP.

- Multi-Sensor Fusion.

- Alpha-Beta Filter.

- Performing AFU.

Taking into account Fingerprinting stage, for every technology $(\lambda)$ considered, the $\mathbf{F}$ matrix must be created. Furthermore, Kernel Density Estimation computational complexity has been proven to be $\mathcal{O}(N L)$ [54]. Therefore, the cost of executing Fingerprinting and TPE is given by $\operatorname{cost}_{T P E}=$ $\Lambda[\mathcal{O}(L)+\mathcal{O}(L N)]$.

Moreover, depending on the architecture pattern choice, the order of the aforementioned MUFAF tasks can change as well as the associated computational cost. In case of TTTF, the computational complexity of solving the separate Kalman Filter for every technology is $\Lambda\left[\mathcal{O}\left(\mathrm{x}^{2}\right)+\mathcal{O}\left(\mathrm{p}^{2}\right)\right]$ where $\mathrm{x}$ and $p$ lengths are 4 and 2 respectively. In case of KFSG, the cost can be expressed as $\mathcal{O}\left((\Lambda \mathbf{x})^{2}\right)$. Additionally, $\alpha-\beta$ filter perform few linear operations: $\mathcal{O}(\mathbf{x})$. Finally, AFU involves the solution of a System of Linear Equations of size $M+1$. Consequently, AFU computational complexity is $\mathcal{O}\left((M+1)^{2}\right)$.

\section{Experiment Results}

The first experiment was run to assess TPE stage. It was executed for a total of 1000 different routes across the room such as the drawn in figure 2. Initially, every route $\Re^{\Lambda}=\left\{\hat{\mathbf{x}}_{1}, \ldots, \hat{\mathbf{x}}_{\varpi=90}\right\}$ was composed of 90 steps. A fingerprint dataset has been created with the cells distribution shown in the scenario figure 2. Measurements were carried out with the available sensors reading simultaneously for a period $\tau=5$ minutes per cell. The number of samples vary according to the technology and distance to the network nodes as previously described in section II. Specifically, for the experiments performed, 28 cells were figured therefore the fingerprint process lasted $28 \times 5=140$ minutes.

The average error for every method is detailed in table II. MUFAF results are significantly better than deterministic methods. In addition, MUFAF results are better than Gaussian Kernel methods except for IEEE802.11.g WiFi sensors. This is probably due to two aspects: (a) distances considered are not long enough to appreciate IEEE 802.11.g WiFi signal 

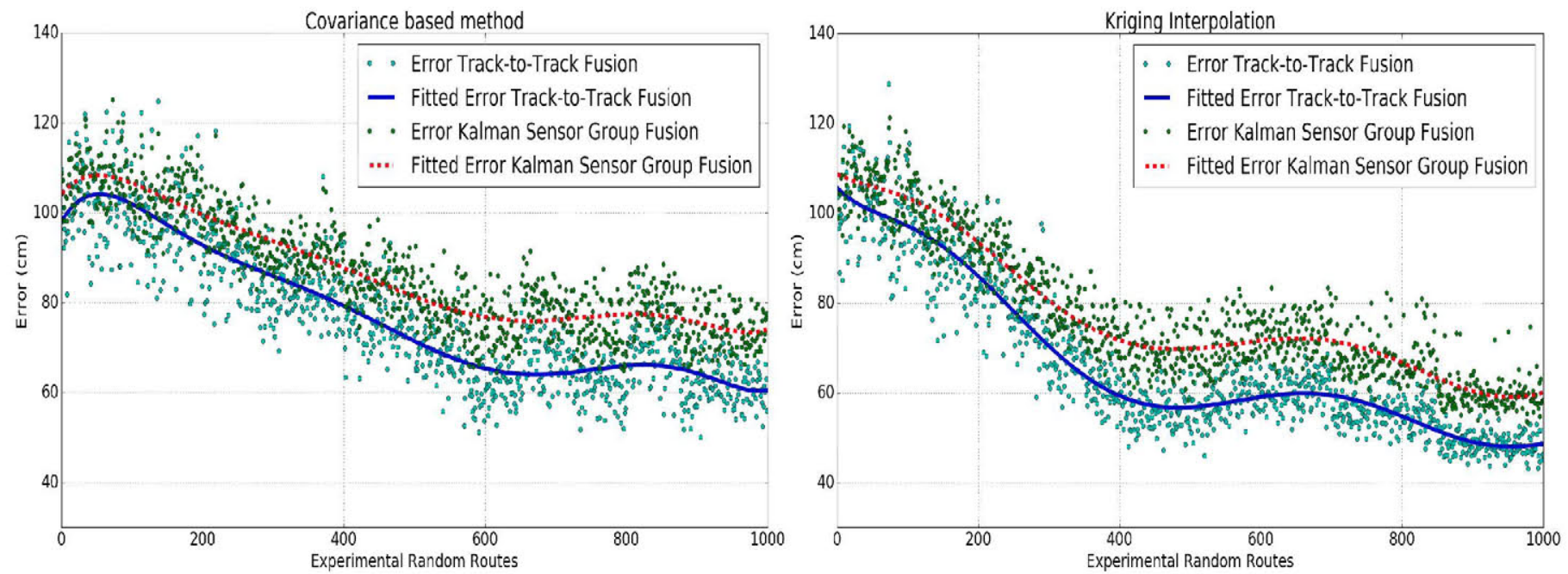

Fig. 10. (Left). Error evolution for Covariance based method in every fusion. (Right). Error evolution for Position Estimation using AFU with a threshold $\mu=1.5$.

attenuation and $(b)$ the histogram distributions are similar to Gaussian functions.

Furthermore, results obtained for the aforementioned sensor technologies are shown in figure 6 . In this figure, it can be observed that IEEE802.15.1 Bluetooth technology errors in estimation are larger than the other technologies. The reasons for this result can be the low power transmission of IEEE 802.15.1 Bluetooth devices and interferences. Conversely, 802.15.4 based technologies perform with a mean error under $70 \mathrm{~cm}$.

Moreover, the architecture patterns described in section $\mathrm{V}$ were also analyzed. In figure 7 , a particular experiment is provided showing the error between the real path and the estimations obtained using the proposed architectures. In the left side, the error variance is large in the initial steps, but it can be observed how this variance is reduced along time due to convergence of $\mathbf{P}$ matrix in $\mathrm{KF}$.

Conversely to expected results, TTTF outperforms KSGF. This can be due to the fact that TTTF employs as much Kalman Filters as technologies $(\lambda)$ available. Therefore, the dimension (range of matrices in KF) is lower than the single one of KSGF. Nonetheless, for a longer assessment period the accuracy of estimations is not significantly enhanced. This is shown in figure 8 , where it can be appreciated that the initial TTTF error is lesser than KSGF, but after some steps in the iteration ( $\geq 100$ steps) it is stabilized. On the other hand, KSGF error is lesser in the long term however, both patterns converge to an error around $45 \mathrm{~cm}$.

Similarly, Adaptive Fingerprinting Update (AFU) was evaluated. The first experiment consisted in comparing Kriging interpolation with Covariance based methods. In the subsequent experiments, the number of steps for each route is 90 . The results are shown in figure 10. In the left side of the figure, MUFAF is tested by using the inverse covariance method [50] whereas the right side of the figure illustrates MUFAF using Kriging interpolation. It can be observed that the method proposed in this paper converges faster to a minimum error value. On the one hand, blue line shows the fitting model that

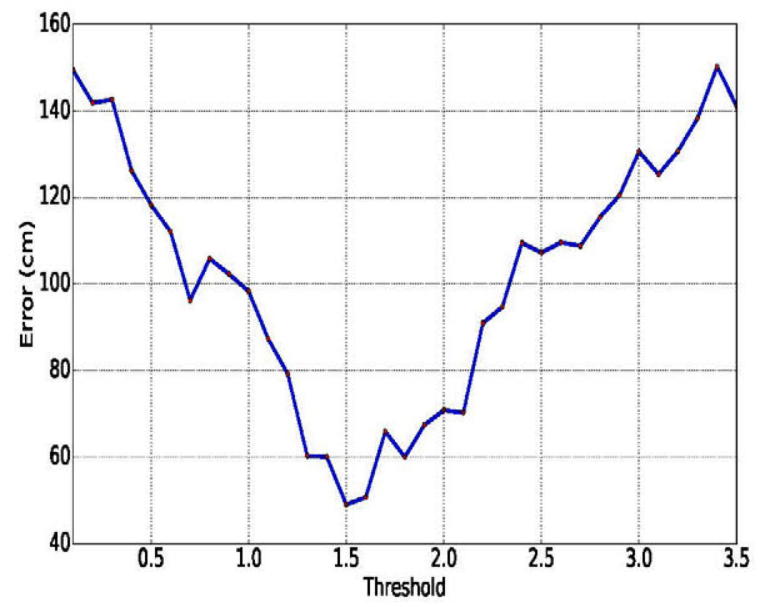

Fig. 11. Empirical threshold for decision rule in AFU. This parameter was obtained by performing iterative simulations for 1000 routes with multiple $\mu$ values in the range $(0,3.5)$.

minimizes the minimum square error (MSE) of estimations for TTTF. On the other hand, red dotted line describes the model that minimizes MSE for KSGF. Results show that MUFAF mitigates the impact of changing conditions. Additionally, the accuracy after some hundred steps is at least between $10-15 \mathrm{~cm}$ better.

In order to evaluate the evolution of the distribution, an experiment consisting in an initial Fingerprinting containing only 50 measurements per cell and technology is performed. Firstly, in figure 9 the evolution of histograms through AFU is illustrated. In top figures, the initial samples distribution is shown while in bottom figures the evolution of distribution after 1000 samples is displayed.

However, one of the most relevant aspects for AFU is the threshold definition. An experiment has been carried out to obtain the performance of AFU applying several values to $\mu$. The results are depicted in figure 11, where the average error for 1000 routes with fixed $\mu$ values show that the optimal value 


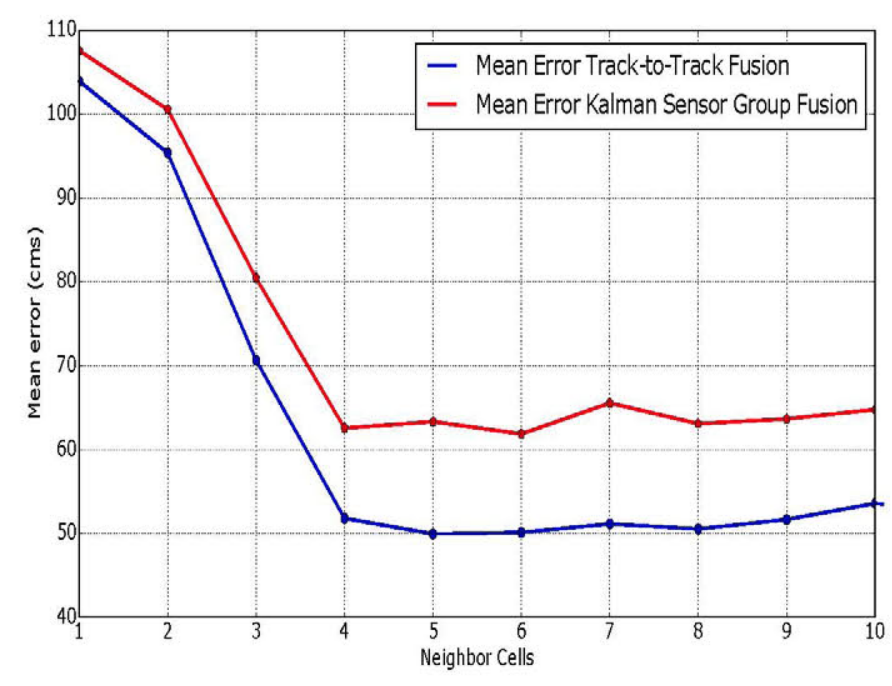

Fig. 12. Mean error expressed in centimeters for Kriging algorithm varying the number of neighbors chosen for interpolation in 1000 routes.

TABLE III

EVOLUTION OF AVERAGE ERROR ( $\mathrm{cms}$ ) OF MUFAF FOR SIMULTANEOUS MULTI-PERSON TRACKING.

\begin{tabular}{|c|c|c|c|c|c|}
\hline $\begin{array}{c}\text { Number } \\
\text { People } \\
\text { Tracked }\end{array}$ & 50 & 100 & 200 & 300 & 400 \\
\hline 1 & 105.56 & 100.35 & 91.09 & 73.01 & 59.67 \\
\hline 2 & 100.96 & 99.01 & 72.48 & 58.76 & 52.87 \\
\hline 3 & 102.62 & 95.03 & 68.10 & 57.85 & 55.12 \\
\hline 4 & 105.64 & 100.47 & 78.66 & 66.28 & 62.02 \\
\hline 5 & 109.35 & 99.39 & 86.59 & 77.33 & 73.60 \\
\hline
\end{tabular}

is $1.3 \leq \mu \leq 1.8$.

Furthermore, AFU influential parameters were analyzed. As stated in previous section VI, the size of the neighbors in Kriging estimation can affect the performance. Therefore a test to show the evolution of $M$ was carried out for both architectures. The results are depicted in figure 12 where the range from $M=\{4,8\}$ yields to the lower error. For large number of cells, the interpolation relevant are not attained. This can be due to the low similarity of the nodes located far away from the estimated point.

Finally, an experiment with several persons randomly walking across the room was performed. Every person held in their hands a device equipped with the technologies mentioned in this work. The fingerprint dataset was initially composed of 50 samples per cell. The results of this test are drawn in table III. Surprisingly, for a single person, the estimation accuracy is lower than multi-person cases. This is mainly due to the faster fingerprint update when the measurements gathered is doubled. However, results for 3 and 4 persons walking simultaneously is similar. The decision threshold $\mu$ allows update the fingerprinting dataset only for high correlated estimations. Additionally, the unexpected multipath, reflection and related events make the variance of fingerprinting dataset to increase according to the number of active users.

The entire dataset containing fingerprinting measurements for every technology, as well as example routes are totally available for testing purposes and comparison with the algorithms described here. This information can be download from http://www.gatv.ssr.upm.es/ $\sim g h p /$

\section{CONCLUSIONS AND FUtURE WORK}

In this paper, a complete framework for accurate indoor tracking has been described. MUFAF is composed by several algorithms that allow to adaptively track an object in an indoor scenario. Furthermore, MUFAF takes advantage of the multiple information sources by means of a fusion strategy. Finally, AFU guarantees the most updated information in the fingerprint data. As an evidence of results obtained, it can be concluded that the fingerprint technique in combination with probabilistic techniques such as Kernels outperform the results obtained by employing deterministic techniques.

Moreover, in the view of results obtained, it can be concluded that the Track-to-Track Fusion strategy outperforms Kalman Sensor Group Fusion for short estimation periods. However, for mid-term applications KSGF can be the most appropriate choice. Up to the best of our knowledge, Kriging interpolation has not been ever used for Adaptive Fusion Update and in this work has been demonstrated that this technique can be successfully employed. The potential application of this technique for statistical estimation such as the KDE has been proven.

As next steps in this Framework development, it is important to highlight the need of improving the process to estimate the $\mu$ parameter, or even to develop techniques that allow to endow it with adaptability to the environment conditions. Furthermore, the employment of advance filtering techniques such as Particle Filters can help to increase the efficiency and precision of estimations performed. Finally, in general terms, technologies employed in this work can be fused to improve the accuracy of estimations.

As future work, this algorithm can be used in combination with computer vision techniques to unequivocally identify people in an indoor environment. Person identification for trackers in Computer Vision is a major issue that can be faced by fusing images information with data gathered from the technologies presented in this work. Additionally, the inclusion of inertial sensor can significantly improve the estimations using absolute orientation values. Moreover, AFU can be analyzed by applying Kriging variants such as Universal Kriging that can be applied to non-stationary fields.

\section{ACKNOWLEDGMENT}

This work was supported by the European Project: ICT4Life http://ict4life.eu/ Grant no. 690090 within the H2020 Research and Innovation Programme.

\section{REFERENCES}

[1] F. De Cillis, F. De Simio, L. Faramondi, F. Inderst, F. Pascucci and R. Setola, "Indoor positioning system using walking pattern classification," Control and Automation (MED), 2014 22nd Mediterranean Conference of, Palermo, 2014, pp. 511-516.

[2] A. Yaeli et al., "Understanding customer behavior using indoor location analysis and visualization," in IBM Journal of Research and Development, vol. 58, no. 5/6, pp. 3:1-3:12, Sept.-Nov. 2014. 
[3] G. Tang et al., "Active tracking using color silhouettes for indoor surveillance," Wireless Communications \& Signal Processing (WCSP), 2015 International Conference on, Nanjing, 2015, pp. 1-5.

[4] C. Xu, B. Firner, Y. Zhang, R. Howard and J. Li, "Poster abstract: Exploiting human mobility trajectory information in indoor device-free passive tracking,"Information Processing in Sensor Networks (IPSN), 2012 ACM/IEEE 11th International Conference on, Beijing, 2012, pp. 121-122.

[5] P. C. Liang, P. Krause, "Real-time indoor patient movement pattern telemonitoring with one-meter precision," Wireless Mobile Communication and Healthcare (Mobihealth), 2014 EAI 4th International Conference on, Athens, 2014, pp. 141-144.

[6] J. A. Kirkup, D. D. Rowlands, D. V. Thiel, "Team Player Tracking Using Sensors and Signal Strength for Indoor Basketball," in IEEE Sensors Journal, vol, 16, no. 11, pp. 4622-4630, June1, 2016.

[7] H. Cho, W. Chung, "Preliminary research on robust leg-tracking indoor mobile robots by combining the Kinect and the laser range finder information," Ubiquitous Robots and Ambient Intelligence (URAI), 2015 12th International Conference on, Goyang, 2015, pp. 462-464

[8] C. Nielsen, J. Nielsen and V. Dehghanian, "Fusion of security camera and RSS fingerprinting for indoor multi-person tracking," 2016 International Conference on Indoor Positioning and Indoor Navigation (IPIN), Alcala de Henares, 2016, pp. 1-7.11

[9] M. Hedley, D. Humphrey, P. Ho, R. Shuttleworth, C. Mackintosh, "Accurate tracking using TOA in sensor networks," Intelligent Sensors, Sensor Networks and Information Processing, 2008. ISSNIP 2008. International Conference on, Sydney, NSW, 2008, pp. 73-78.

[10] H. Xiong, Z. Chen, B. Yang, R. Ni, emph"TDOA localization algorithm with compensation of clock offset for wireless sensor networks," in China Communications, vol. 12, no. 10, pp. 193-201, Oct. 2015.

[11] F. Wen, C. Liang, "An Indoor AOA Estimation Algorithm for IEEE 802.11ac Wi-Fi Signal Using Single Access Point," in IEEE Communications Letters, vol. 18, no. 12, pp. 2197-2200, Dec. 2014.

[12] W. Ruan, Q. Z. Sheng, L. Yao, T. Gu, M. Ruta, L. Shangguan, "Device-free indoor localization and tracking through Human-Object Interactions," 2016 IEEE 17th International Symposium on A World of Wireless, Mobile and Multimedia Networks (WoWMoM), Coimbra, 2016, pp. 1-9.

[13] S. Mahfouz, F. Mourad-Chehade, P. Honeine, J. Farah and H. Snoussi, "Non-Parametric and Semi-Parametric RSSI/Distance Modeling for Target Tracking in Wireless Sensor Networks," in IEEE Sensors Journal, vol. 16, no. 7, pp. 2115-2126, April 1, 2016.

[14] M. H. Habaebi, R. O. Khamis, A. Zyoud, M. R. Islam, "RSS Based Localization Techniques for ZigBee Wireless Sensor Network," Computer and Communication Engineering (ICCCE), 2014 International Conference on, Kuala Lumpur, 2014, pp. 72-75.

[15] Z. Li, D. B. Acua, Z. Zhao, J. L. Carrera, T. Braun, "Fine-grained indoor tracking by fusing inertial sensor and physical layer information in WLANs," 2016 IEEE International Conference on Communications (ICC), Kuala Lumpur, 2016, pp. 1-7.

[16] H. Yang; R. Zhan, J. Bordoy, F. Hoflinger, W. Li, C. Schindelhauer, L. Reindl, "Smartphone based integrated Indoor Localization System using Inertial Sensor and Acoustic Transmitter/Receiver," in IEEE Sensors Journal, vol.PP, no.99, pp.1-1.

[17] S. Cai, W. Liao, C. Luo, M. Li, X. Huang, P. Li, "CRIL: An Efficient Online Adaptive Indoor Localization System," in IEEE Transactions on Vehicular Technology, vol.PP, no.99, pp.1-1.

[18] H. Hashemi, "The indoor radio propagation channel" in Proceedings of the IEEE, vol. 81, no. 7, pp. 943-968, Jul 1993

[19] X. He, J. Li, D. Aloi, "WiFi based indoor localization with adaptive motion model using smartphone motion sensors," 2014 International Conference on Connected Vehicles and Expo (ICCVE), Vienna, 2014, pp. 786-791.

[20] S. Kokalj-Filipovic, L. Greenstein, B. Cheng and M. Gruteser, "Methods for Extracting V2V Propagation Models from Imperfect RSSI Field Data," Vehicular Technology Conference (VTC Fall), 2015 IEEE 82nd, Boston, MA, 2015, pp. 1-5.

[21] S. Yiu, M. Dashti, H. Claussen, F. Perez-Cruz, "Locating user equip ments and access points using RSSI fingerprints: A Gaussian process approach," 2016 IEEE International Conference on Communications (ICC), Kuala Lumpur, 2016, pp. 1-6.

[22] J. Y. Zhu, A. X. Zheng, J. Xu, V. O. K. Li, "Spatio-temporal (S-T) similarity model for constructing WIFI-based RSSI fingerprinting map for indoor localization," Indoor Positioning and Indoor Navigation (IPIN), 2014 International Conference on, Busan, 2014, pp. 678-684.

[23] S. Doan, F. Ylmaz, S. Ertrk, "On the error performance bounds of positioning using RSSI-based fingerprints," 2015 23nd Signal Processing and Communications Applications Conference (SIU), Malatya, 2015, pp $1325-1328$

[24] JooSeuk Kim, C. Scott, "Robust kemel density estimation," 2008 IEEE International Conference on Acoustics, Speech and Signal Processing, Las Vegas, NV, 2008, pp. 3381-3384.

[25] V. Akbarzadeh, C. Gagn, M. Parizeau, "Kernel density estimation for target trajectory prediction," Intelligent Robots and Systems (IROS) 2015 IEEE/RSJ International Conference on, Hamburg, 2015, pp. 34493456

[26] A. Kushki, K.N. Plataniotis, A.N. Venetsanopoulol, C. S. Regazzoni, "Radio Map Fusion for Indoor Positioning in Wireless Local Area Networks," 2005 7th International Conference on Information Fusion (FUSION).

[27] J. Yim, S. Jeong, J. Joo, C. Park, "Utilizing Map Information for WLANBased Kalman Filter Indoor Tracking," Future Generation Communication and Networking Symposia, 2008. FGCNS '08. Second International Conference on, Sanya, 2008, pp. 58-62.

[28] S. Feng. R. Murray-Smith, "Fusing Kinect sensor and inertial sensors with multi-rate Kalman filter," Data Fusion \& Target Tracking 2014: Algorithms and Applications (DF\&TT 2014), IET Conference on, Liverpool, UK, 2014, pp. 1-8.

[29] Ngoc-Huyn Ho, Phuc Huu Truong, and Gu-Min Jeong, "Step-Detection and Adaptive Step-Length Estimation for Pedestrian Dead-Reckoning at Various Walking Speeds Using a Smartphone.", Sensors (Basel). 2016 Sep: 16(9): 1423. Published online 2016 Sep 2. doi: 10.3390/s16091423.

[30] Farzin Dadashi, Florent Crettenand,Grgoire P. Millet, and Kamiar Aminian, "Front-Crawl Instantaneous Velocity Estimation Using a Wearable Inertial Measurement Unit', Sensors (Basel). 2012; 12(10): 1292712939. Published online 2012 Sep 25. doi: 10.3390/s121012927

[31] K. Lu, K. C. Chang, R. Zhou, "The exact algorithm for multi-sensor asynchronous track-to-track fusion," Information Fusion (Fusion), 2015 18th International Conference on, Washington, DC, 2015, pp. 886-892.

[32] Pengfei Guo, Xiaogang Wang, Yuehua Wu, "Data Fusion Using Weighted Likelihood," European Journal of Pure and Applied Mathematics Vol. 5, No. 3, 2012, pp. 333-356.

[33] E. Mirzazadeh, B. Moshiri, "Multi-sensor estimation using CKF and DKF,"Computer and Knowledge Engineering (ICCKE), 2013 3th International eConference on, Mashhad, 2013, pp. 321-325.

[34] Sayed Amir Hoseini, Mohammad Reza Ashraf, "Computational Complexity Comparison of Multi-sensor Single Target Data Fusion Methods by Matlab", International Journal of Chaos, Control, Modelling and Simulation (IJCCMS) Vol.2, No.2, June 2013

[35] Dirk Tenne, Tarunraj Singh, "Optimal Design of $\alpha-\beta-\gamma$ Filters," American Control Conference, 2000. Proceedings of the 2000 (Volume:6), pp 43484352

[36] C. Liu, A. Kiring, N. Salman, L. Mihaylova, I. Esnaola, "A Kriging algorithm for location fingerprinting based on received signal strength," Sensor Data Fusion: Trends, Solutions, Applications (SDF), 2015, Bonn, 2015, pp. 1-6.

[37] Gustavo Hernandez-Penaloza, Baltasar Beferull-Lozano, "Field Estimation in Wireless Sensor Networks Using Distributed Kriging," IEEE ICC 2012 - Ad-hoc and Sensor Networking Symposium.

[38] Shau-Shiun Jan, Shuo-Ju Yeh, Ya-Wen Liu, "Received Signal Strength Database Interpolation by Kriging for a Wi-Fi Indoor Positioning System," Sensors 2015, 15(9), 21377-21393.

[39] Tareq Alhmiedat, Ghassan Samara, Amer O. Abu Salem, "An Indoor Fingerprinting Localization Approach for ZigBee Wireless Sensor Networks," European Journal of Scientific Research Vol. 105 No 2 July, 2013, pp.190-202.

[40] Schucany, William R., Kernel Smoothers: An Overview of Curve Estimators for the First Graduate Course in Nonparametric Statistics Statist. Sci. 19 (2004), no. 4, 663-675.

[41] Arsham Farshad, Jiwei Li, Mahesh K. Marina, Francisco J. Garcia, "A Microscopic Look at WiFi Fingerprinting for Indoor Mobile Phone Localization in Diverse Environments" 2013 International Conference on Indoor Positioning and Indoor Navigation, 28-31st October 2013.

[42] Niu, J., Wang, B., Shu, L., Duong, T. Q., Chen, "ZIL: An EnergyEfficient Indoor Localization System Using ZigBee Radio to Detect WiFt Fingerprints," IEEE Journal on Selected Areas in Communications, 33(7), pp. 1431-1442

[43] Beomju Shin. Jung Ho Lee, Taikjin Lee, Hyung Seok Kim, "Enhanced Weighted K-Nearest Neighbour Algorithm for Indoor Wi-Fi Positioning Systems," 2012 8th International Conference on Volume 2, pp. 574577.

[44] Vahid Fathabadi, Mehdi Shahbazian, Karim Salahshour, Lotfollah Jargani. Comparison of Adaptive Kalman Filter Methods in State Estimation of a Nonlinear System Using Asynchronous Measurements. Proceedings of the World Congress on Engineering and Computer Science 2009. 
[45] Pengfei Guo, Xiaogang Wang, Yuehua Wu, "Data Fusion Using Weighted Likelihood," European Journal of Pure and Applied Mathematics Vol. 5, No. 3, 2012, pp. 333-356.

[46] G. Hernandez-Peñaloza, C. Asensio-Marco and B. Beferull-Lozano, "Distributed estimation of statistical correlation measures for spatial inference in WSNs"Signal Processing Conference (EUSIPCO), 2012 Proceedings of the 20th European, Bucharest, 2012, pp. 699-703.

[47] Y. s. Chiou, C. 1. Wang and S. C. Yeh, "Reduced-complexity scheme using alpha-beta filtering for location tracking" ET Communications, vol. 5, no. 13, pp. 1806-1813, Sept. 52011.

[48] David M. Bashtannyk, Rob J. Hyndman, "Bandwidth selection for kernel conditional density estimation" Computational Statistics and Data Analysis 36 (2001) 279298.

[49] Kechen Zheng, Jingjing Luo, Jinbei Zhang, Weijie Wu, Xiaohua Tian, Xinbing Wang, "Cooperation Improves Delay in Cognitive Networks With Hybrid Random Walk" Communications IEEE Transactions on, vol. 63, pp. 1988-2000, 2015, ISSN 0090-6778.

[50] Ian L. Dryden, Alexey Koloydenko and Diwei Zhou, Non-Euclidean Statistics for Covariance Matrices, with Applications to Diffusion Tensor Imaging. The Annals of Applied Statistics Vol. 3, No. 3 (Sep., 2009), pp. 1102-1123.

[51] Bluetooth technology, https://www.bluetooth.com/develop-with-bluetoo th

[52] IEEE802.15.4 WPAN Task group 4, http://www.ieee802.org/15/pub/TG 4. $\mathrm{html}$

[53] IEEE 802.11 Wireless Local Area Networks, http://www.ieee802.org/11/

[54] Lasse Holmstrm, The Accuracy and the Computational Complexity of a Multivariate Binned Kernel Density Estimator, Journal of Multivariate Analysis, Volume 72, Issue 2, February 2000, Pages 264-309, ISSN 0047259X, http://dx.doi.org/10.1006/jmva.1999.1863. 